\title{
Gegenbauer Transformations Nikolski-Besov Spaces Generalized by Gegenbauer Operator and Their Approximation Characteristics
}

\author{
V.S. Guliyev ${ }^{1,2^{*}}$, E.J. Ibrahimov ${ }^{2}$ and S.Ar. Jafarova ${ }^{3}$ \\ ${ }^{1}$ Ahi Evran University, Department of Mathematics, 40100, Kirsehir, Turkey \\ Email: vagif@guliyev.com \\ ${ }^{2}$ Institute of Mathematics and Mechanics, AZ 1141 Baku, Azerbaijan \\ Email: elmanibrahimov@yahoo.com \\ ${ }^{3}$ Azerbaijan State Economic University 6, Istiglaliyyat str., Baku AZ1001, Azerbaijan \\ Email: sada-jafarova@rambler.ru
}

\begin{abstract}
In this paper we consider some problems of the theory of approximation of functions on interval $[0, \infty)$ in the metric of $L_{p, \lambda}$ with weight $s h^{2 \lambda} x$ using generalized Gegenbauer shifts We prove analogues of direct Jackson theorems for the modulus of smoothness of arbitrary order defined in terms of generalized Gegenbauer shifts. We establish the equivalence of the modulus of smoothness and $K$-functional, defined in terms of the space of the Sobolev type corresponding to the Gegenbauer differential operator. We define function spaces of Nikol'skii-Besov type and describe them in terms of best approximations. As a tool for approximation, we use some functions classes of spectrum. In these classes, we prove analogues of Bernstein's inequality and others for the Gegenbauer differential operator. Our results are analogues of the results for generalized Bessel shifts obtained in the work [30].
\end{abstract}

Keywords: Approximation of functions, generalized Gegenbauer shift, Gegenbauer transformation, Nikol'skii-Besov type spaces, embedding theorems.

In the classical theory of approximation of functions on $\mathbb{R}=(-\infty, \infty)$ the shift operator $f(x) \mapsto f(x+y)$, $x, y \in \mathbb{R}$. plays a central role. This shift operator is used in the construction of the moduli of continuity and smoothness, which are the basic elements of the direct and inverse theorems of approximation theory. Various generalizations of shift operators enable to state natural analogues of problems in approximation theory. Groups and semigroups of operators on Banach spaces are generalizations of the shift operator. Different problems of approximation theory on Banach spaces with groups and semigroups of operators were considered in $[1,3,5,7,44]$.

Generalized shift operator naturally follows from "addition theorem" for eigen functions of the differential operators (for example, Legendre, Gegenbauer, Jacobi, Laguerre, Hermite and other). These operators may not form a group or semigroup, but the generalized moduli of smoothness defined in terms of them can be better adapted to the study of relations between the smoothness properties of functions and the best approximations of these functions in weighted function spaces. Some results on the best approximation of functions using generalized shift operators can be found in [1, 4, 22-26, 29-39, 45]. Note that most of the papers on this topic deal with the approximation of functions by polynomials on a finite segment. For the half-line, most popular examples are the generalized Bessel and Dunkl shifts (see for example [4, 23, 30-32]). Fourier-Bessel and Fourier-Dunkl harmonic analysis, which deals with Bessel and Dunkl integral transformations and their applications, are closely connected with the generalized Bessel and Dunkl shift. Moreover, generalized Bessel shift is widely used in the potential theory and theory of maximal functions (see, example [5, 11-13]). The file of constructions of the theory of generalized shift operators generalize in the theory of transformation of operators (see for example [7]). We reduce only these works which have at least some relation to the paper. In this paper the generalized Gegenbauer shift are considered and by using this some questions of approximation theory of functions in $[0, \infty)$ in the metric $L_{p, \lambda}$ with weight $s h^{2 \lambda} x$ are studied. 


\section{The Properties of Generalized Gegenbauer Shift and Statement of Main Results}

For every $p, 1 \leq p<\infty$, we denote by $L_{p, \lambda}$ the Banach space of measurable functions $f$ on $\mathbb{R}_{+}$(defined up to their values on a set of measure zero) such that the norm

$$
\|f\|_{p, \lambda} \equiv\|f(c h)(\cdot)\|_{p, \lambda}=\left(\int_{0}^{\infty}|f(c h t)|^{p} s h^{2 \lambda} t \mathrm{~d} t\right)^{\frac{1}{p}}
$$

is finite.

For $p=\infty$ we denote by $L_{\infty, \lambda}$ the set of all functions $f$ that are uniformly continuous and bounded on $\mathbb{R}_{+}$. The norm in $L_{\infty, \lambda}$ is defined by the formula

$$
\|f\|_{\infty, \lambda} \equiv\|f(\operatorname{ch})(\cdot)\|_{\infty, \lambda}=\sup _{t \in \mathbb{R}_{+}}|f(\operatorname{ch} t)| .
$$

Let

$$
D_{\lambda}=\left(x^{2}-1\right)^{\frac{1}{2}-\lambda} \frac{\mathrm{d}}{\mathrm{d} x}\left(x^{2}-1\right)^{\lambda+\frac{1}{2}} \frac{\mathrm{d}}{\mathrm{d} x}
$$

be Gegenbauer differential operators.

The functions (see [9], for.(2.3) and (2.8), and also [11], p.1045, for. 8.936(1))

$$
\begin{gathered}
P_{\alpha}^{\lambda}(\operatorname{ch} x)=\frac{\Gamma(\alpha+2 \lambda) \cos \lambda \pi}{\Gamma(\lambda) \Gamma(\alpha+\lambda+1)}(2 \operatorname{ch} x)^{-\alpha-2 \lambda} \\
\times F\left(\frac{\alpha}{2}+\lambda, \frac{\alpha}{2}+\lambda+\frac{1}{2} ; \alpha+\lambda+1 ; \frac{1}{c^{2} x}\right), x \geq 0 \\
C_{\alpha}^{\lambda}(\operatorname{ch} x)=\sqrt{\pi} \frac{\Gamma(\alpha+2 \lambda)}{\Gamma(\lambda) \Gamma(\alpha+1)}(2 \operatorname{sh} x)^{\frac{1}{2}-\lambda} P_{\alpha+\lambda-1}^{\frac{1}{2}-\lambda}(\operatorname{ch} x) \\
=\frac{\Gamma(\alpha+\lambda)}{\Gamma(\lambda) \Gamma(\alpha+1)}(2 \operatorname{ch} x)^{\alpha} F\left(-\frac{\alpha}{2},-\frac{\alpha}{2}+\frac{1}{2} ; 1-\alpha-\lambda ; \frac{1}{c^{2} x}\right),
\end{gathered}
$$

where $P_{\alpha+\lambda-1}^{\frac{1}{2}-\lambda}(\operatorname{ch} x)$ is Legendre function, and $F(\alpha, \beta ; \gamma ; x)$ is Gauss's hypergeometric function, are the linearly independent solutions of equation

$$
\operatorname{sh}^{2} x y^{\prime \prime}(\operatorname{ch} x)+(2 \lambda+1) \operatorname{ch} x y^{\prime}(\operatorname{ch} x)-\alpha(\alpha+2 \lambda) y(\operatorname{ch} x)=0 .
$$

For the functions $P_{\alpha}^{\lambda}(\operatorname{ch} x)$ and $C_{\alpha}^{\lambda}(\operatorname{ch} x)$ the following formulas (see [9], p. 1939) are valid:

$$
\begin{gathered}
P_{\alpha}^{\lambda}(\operatorname{ch} x \operatorname{ch} t-\operatorname{sh} x \operatorname{sh} \cos \varphi) \\
=\frac{\Gamma(2 \lambda-1)}{\Gamma^{2}(\lambda)} \sum_{n=0}^{\infty}(-1)^{n} \frac{4^{n} \Gamma(\alpha-n+1) \Gamma^{2}(\lambda+n)(2 n+2 \lambda-1)}{\Gamma(\alpha+2 \lambda+n)} \\
\times \operatorname{sh}^{n} x \operatorname{sh}^{n} t P_{\alpha-n}^{\lambda+n}(\operatorname{ch} x) C_{\alpha-n}^{\lambda+n}(\operatorname{cht}) C_{n}^{\lambda-\frac{1}{2}}(\cos \varphi), \\
=\frac{\Gamma(2 \lambda-1)}{\Gamma^{2}(\lambda)} \sum_{n=0}^{\infty}(-1)^{n} \frac{4^{n} \Gamma(\alpha-n+1) \Gamma^{2}(\alpha+n)(2 n+2 \lambda-1)}{\Gamma(a+2 \lambda+n)} \\
\times \operatorname{sh}^{n} x \operatorname{sh}^{n} t C_{\alpha-n}^{\lambda+n}(\operatorname{ch} x) C_{\alpha-n}^{\lambda+n}(\operatorname{cht} t) C_{n}^{\lambda-\frac{1}{2}}(\cos \varphi),
\end{gathered}
$$

where $\alpha-n \neq-1,-2, \ldots$. 
Taking into account the following equation (see [11], p.844)

$$
\int_{0}^{\pi} C_{n}^{\lambda-\frac{1}{2}}(\cos \varphi)(\sin \varphi)^{2 \lambda-1} \mathrm{~d} \varphi= \begin{cases}0, & n \geq 1, \\ \frac{\Gamma(\lambda) \Gamma\left(\frac{1}{2}\right)}{\Gamma\left(\lambda+\frac{1}{2}\right)}, & n=0\end{cases}
$$

from (1.5) and (1.6) we obtain

$$
\begin{gathered}
A_{c h t}^{\lambda} P_{\alpha}^{\lambda}(\operatorname{ch} x)=\frac{\Gamma\left(\lambda+\frac{1}{2}\right)}{\Gamma(\lambda) \Gamma\left(\frac{1}{2}\right)} \int_{0}^{\pi} P_{\alpha}^{\lambda}(\operatorname{ch} x \operatorname{cht}-\operatorname{sh} x \operatorname{sht} \cos \varphi)(\sin \varphi)^{2 \lambda-1} \mathrm{~d} \varphi \\
=\frac{\Gamma(2 \lambda) \Gamma(\alpha+1)}{\Gamma(\alpha+2 \lambda)} P_{\alpha}^{\lambda}(\operatorname{cht}) C_{\alpha}^{\lambda}(\operatorname{ch} x)=P_{\alpha}^{\lambda}(\operatorname{cht}) Q_{\alpha}^{\lambda}(\operatorname{ch} x), \\
A_{c h t}^{\lambda} Q_{\alpha}^{\lambda}(\operatorname{ch} x)=\frac{\Gamma\left(\lambda+\frac{1}{2}\right)}{\Gamma(\lambda) \Gamma\left(\frac{1}{2}\right)} \int_{0}^{\pi} Q_{\alpha}^{\lambda}(\operatorname{ch} x \operatorname{cht}-\operatorname{sh} x \operatorname{sht} \cos \varphi)(\sin \varphi)^{2 \lambda-1} \mathrm{~d} \varphi \\
=Q_{\alpha}^{\lambda}(\operatorname{cht}) Q_{\alpha}^{\lambda}(\operatorname{ch} x) .
\end{gathered}
$$

In (1.7) and (1.8)

$$
Q_{\alpha}^{\lambda}(\operatorname{ch} x)=\frac{\Gamma(2 \lambda) \Gamma(\alpha+1)}{\Gamma(\alpha+2 \lambda)} C_{\alpha}^{\lambda}(\operatorname{ch} x)
$$

where

$$
\begin{gathered}
A_{c h t}^{\lambda} f(\operatorname{ch} x) \equiv A_{\operatorname{cht}} f(\operatorname{ch} x) \\
=C(\lambda) \int_{0}^{\pi} f(\operatorname{ch} x \operatorname{cht}-\operatorname{sh} x \operatorname{sht} \cos \varphi)(\sin \varphi)^{2 \lambda-1} \mathrm{~d} \varphi
\end{gathered}
$$

and

$$
C(\lambda)=\frac{\Gamma\left(\lambda+\frac{1}{2}\right)}{\Gamma(\lambda) \Gamma\left(\frac{1}{2}\right)}=\left(\int_{0}^{\pi}(\sin \varphi)^{2 \lambda-1} \mathrm{~d} \varphi\right)^{-1}
$$

are generalized Gegenbauer shift operator, follows from "addition theorem" (1.5) and (1.6) for Gegenbauer functions naturally.

Here and further after $c(a, b, \ldots)>0$ will denote the constants, dependent only on written parameter, moreover this perhaps differs in different of formulas.

The generalized Gegenbauer shift operator possesses some properties which are analogues to generalized Bessel shift operator in the work of Levitan [26]:

1) Linearity and homogeneity:

$$
A_{\text {ch } t}\{a f(\operatorname{ch} x)+b g(\operatorname{ch} x)\}=a A_{\text {ch } t} f(\operatorname{ch} x)+b A_{\text {ch } t} g(\operatorname{ch} x),
$$

which follows from the integral property.

2) Positivity. $A_{\text {ch } t} f(\operatorname{ch} x) \geq 0$, if $f(\operatorname{ch} x) \geq 0$, which is evident.

3) $A_{1} f(\operatorname{ch} x)=f(\operatorname{ch} x), A_{\text {ch } t} 1=1$, which follows from (1.9).

4) If $f(\operatorname{ch} x) \equiv 0$ for $x \geq a$, then $A_{\text {ch } t} f(\operatorname{ch} x) \equiv 0$ for $|x-t| \geq a$.

Indeed, ch $x$ ch $t-\operatorname{sh} x \operatorname{sh} t \cos \varphi \geq \operatorname{ch}(x-t) \geq|x-t|$, from this the property 4) follows.

5) $L_{p, \lambda}$-boundedness. For any $f \in L_{p, \lambda}, 1 \leq p \leq \infty$, (see [14], Lemma 2)

$$
\left\|A_{c h t} f\right\|_{p, \lambda} \leq\|f\|_{p, \lambda}, \quad t \in[0, \infty) .
$$

6) The operator $A_{c h t}$ is continuous on $L_{p, \lambda}$.

7) Symmetry of the operator $A_{c h t}$,

$$
A_{\text {ch } t} f(\operatorname{ch} x)=A_{\operatorname{ch} x} f(\operatorname{cht})
$$

is evident. 
8) Commutativity. For every continuous functions $f(\operatorname{ch} x)$ and any really $y, t \geq 0$ the following equality is valid:

$$
A_{\text {ch } y} A_{\operatorname{ch} t} f(\operatorname{ch} x)=A_{\operatorname{ch} t} A_{\operatorname{ch} y} f(\operatorname{ch} x) .
$$

9) The operator $A_{c h t}$ is self-adjoint (see [14], Lemma 3). This property is proved in [13].

For any $f, g \in L_{1, \lambda}$ the following equality is valid:

$$
\int_{0}^{\infty} A_{\operatorname{cht} t} f(\operatorname{ch} x) g(\operatorname{ch} t) \operatorname{sh}^{2 \lambda} t \mathrm{~d} t=\int_{0}^{\infty} f(\operatorname{ch} t) A_{\operatorname{cht} t} g(\operatorname{ch} x) \operatorname{sh}^{2 \lambda} t \mathrm{~d} t .
$$

For every $f \in L_{p, \lambda}$ the differences of order $k(k=1,2, \ldots)$ are defined by the formula

$$
\begin{gathered}
\Delta_{\text {ch } t}^{1} f(\operatorname{ch} x)=\Delta_{\text {cht }} f(\operatorname{ch} x):=A_{\text {ch } t} f(\operatorname{ch} x)-f(\operatorname{ch} x), \\
\Delta_{\text {cht }}^{k} f(\operatorname{ch} x):=A_{\text {ch } t}\left(\Delta_{\text {ch } t}^{k-1} f(\operatorname{ch} x)\right), k=2,3, \ldots
\end{gathered}
$$

or

$$
\Delta_{c h t}^{k} f(\operatorname{ch} x):=\sum_{\nu=0}^{k}(-1)^{k-\nu}\left(\begin{array}{l}
k \\
\nu
\end{array}\right) A_{c h t}^{\nu} f(\operatorname{ch} x) .
$$

For every natural number $k$ the modulus $\omega_{k}(f, \delta)_{p, \lambda}$ of order $k$ in $L_{p, \lambda}$ are defined by the formula

$$
\omega_{k}(f, \delta)_{p, \lambda}:=\sup _{0<t \leq \delta}\left\|\Delta_{c h t}^{k} f\right\|_{p, \lambda}
$$

We say that the function $f \in L_{p, \lambda}$ has bounded spectrum of order $\nu$, if $\hat{f}_{p}(\alpha)=0$ for $\alpha>\nu$ (the definition $\hat{f}_{p}(\alpha)$ see lower the formula $\left.(2.1)\right)$.

We denote by $D_{\lambda}^{k}[0, \infty)$ the class of functions which we apply the operator $D_{\lambda}$ to them $k$-times, i.e., $\exists D_{\lambda}^{k} f, k=1,2, \ldots$.

Let $W_{p, \lambda}^{m}, m=1,2, \ldots$, be the Sobolev space constructed for the differential operator $D_{\lambda}$, that is

$$
W_{p, \lambda}^{m}:=\left\{f \in L_{p, \lambda}: D_{\lambda}^{k} f \in L_{p, \lambda}, k=1,2, . ., m\right\} .
$$

We denote by $\mathfrak{M}(\nu, p, \lambda), \nu>0$ the set of functions $\Phi(\operatorname{ch} x), x \in \mathbb{R}$, which satisfies the following conditions:

1) $\Phi(\operatorname{ch} x)$ is the spectrum of order $\nu$;

2) $\Phi(\operatorname{ch} x)$ belongs to the class $W_{p, \lambda}^{m}$.

The best approximation of an $f \in L_{p, \lambda}$ by functions belonging to $\mathfrak{M}(\nu, p, \lambda)$ is defined as follows:

$$
E_{\nu}(f):=\inf \left\{\|f-\Phi\|_{p, \lambda}: \Phi \in \mathfrak{M}(\nu, p, \lambda)\right\}
$$

The symbol $0 \leq a \lesssim b$ means that $a \leq c b$, where $c$-some constant, which can dependent on some parameters. The following theorem is an analogue of Jackson's direct theorem in classical approximation theory (see [41], ch. 5).

Theorem 1.1. Let $f \in W_{p, \lambda}^{m}$. Then for all $\nu>0$ and $m \in \mathbb{Z}_{+}=\{0,1,2, \ldots\}$, the inequality

$$
E_{\nu}(f)_{p, \lambda} \lesssim \nu^{-2 m} \omega_{k}\left(D_{\lambda}^{m} f, \frac{1}{v}\right)_{p, \lambda}
$$

holds.

We define the $K$-functional Peetre construction for $L_{p, \lambda}$ and $W_{p, \lambda}^{m}$ by the formula:

$$
K\left(f, t ; L_{p, \lambda} ; W_{p, \lambda}^{m}\right):=\inf \left\{\|f-g\|_{p, \lambda}+t\left\|D_{\lambda}^{m} g\right\|_{p, \lambda}: g \in W_{p, \lambda}^{m}\right\},
$$

where $f \in L_{p, \lambda}$ and $t>0$.

The symbol $\alpha(t) \simeq \beta(t)$ at $t \rightarrow 0$ means that there exists constants $c_{1}$ and $c_{2}$ such that

$$
c_{1} \alpha(t) \leq \beta(t) \leq c_{2} \alpha(t)
$$


The next theorem shows that the modulus of smoothness is equivalent to the $K$-functional.

Theorem 1.2. For every $\delta>0$ the following correlation

$$
K\left(f, \delta^{2 m} ; L_{p, \lambda} ; W_{p, \lambda}^{m}\right) \simeq \omega_{m}(f, \delta)_{p, \lambda}, \quad t \rightarrow 0
$$

holds.

The proofs of inverse theorems in approximation theory are based on various analogues of Bernstein's inequality. In this paper we obtain such analogues for the Gegenbauer differential operator $D_{\lambda}$.

Theorem 1.3. The following inequality is valid:

$$
\left\|D_{\lambda} f\right\|_{p, \lambda} \leq \nu^{2}\|f\|_{p, \lambda}
$$

for all $f \in \mathfrak{M}(\nu, p, \lambda)$.

Inequality (1.16) is an analogue of the classical Bernstein inequality for entire functions of exponential type (see [28], p.117).

Let $r>0$ be a real number and let $k, m$ be non-negative numbers such that $2 k>r-2 m>0$. We denote by $H_{p, \lambda}^{r}$ the set of all $f \in W_{p, \lambda}^{m}$ such that and

$$
\omega_{k}\left(D_{\lambda}^{m}, \delta\right)_{p, \lambda} \leq A_{f} \delta^{r-2 m}, \delta>0
$$

for some $A_{f}>0$. For $f \in H_{p, \lambda}^{r}$ we define the seminorm as

$$
h_{p, \lambda}^{r}(f):=\sup _{\delta>0} \frac{\omega_{k}\left(D_{\lambda}^{m}, \delta\right)_{p, \lambda}}{\delta^{r-2 m}} .
$$

$H_{p, \lambda}^{r}$ is a Banach space with norm (see section 5)

$$
\|f\|_{H_{p, \lambda}^{r}}:=\|f\|_{p, \lambda}+h_{p, \lambda}^{r}(f) .
$$

In the next theorem we describe the space $H_{p, \lambda}^{r}$ in terms of approximations by functions belonging to $\mathfrak{M}(\nu, p, \lambda)$. In particular, this theorem implies that the $H_{p, \lambda}^{r}$ does not depend on $k$ or $m$.

Theorem 1.4. If $f \in H_{p, \lambda}^{r}$, then

$$
E_{\nu}(f)_{p, \lambda} \lesssim \nu^{-r} h_{p, \lambda}^{r}(f)
$$

for $\nu \geq 1$.

Conversely, if $f \in L_{p, \lambda}$, then

$$
E_{\nu}(f)_{p, \lambda} \leq \frac{A_{f}}{\nu^{r}}
$$

for $\nu \geq 1$, where $A_{f}$ is a constant depending on $f$.

Let $1 \leq q \leq \infty, r>0$, and let $k, m$ be non-negative integers such that $2 k>r-2 m>0$. As in [27], we say that a function $f$ belongs to the Nikol'skii-Besov class $B_{p, q, \lambda}^{r} \equiv B_{p, q, \lambda}^{r}\left(\mathbb{R}_{+}\right)$if $f \in W_{p, \lambda}^{m}$ and the seminorm

$$
b_{p, q, \lambda}^{r}(f):=\left\{\begin{array}{l}
\left(\int_{0}^{\infty} \frac{\left(\omega_{k}\left(D_{\lambda}^{m} f, \delta\right)_{p, \lambda}\right)^{q}}{\delta^{(r-2 m) q}} \frac{\mathrm{d} \delta}{\delta}\right)^{\frac{1}{q}}, 1 \leq q<\infty, \\
\sup _{\delta>0} \frac{\omega_{k}\left(D_{\lambda}^{m} f, \delta\right)_{p, \lambda}}{\delta^{r-2 m}}, \quad q=\infty
\end{array}\right.
$$

is finite. Note that $B_{p, q, \lambda}^{r}$ is a Banach space with norm (see section 5)

$$
\|f\|_{B_{p, q, \lambda}^{r}}:=\|f\|_{p, \lambda}+b_{p, q, \lambda}^{r} .
$$

Note that $B_{p, \infty, \lambda}^{r}=H_{p, \lambda}^{r}$. Let $\mathbb{Z}_{+}=\{0,1,2, \ldots\}$. 
Theorem 1.5. Let $a>1$ be an arbitrary number. The function $f \in L_{p, \lambda}$ belongs to $B_{p, q, \lambda}^{r}$ if and only if the seminorm

$$
\tilde{b}_{p, q, \lambda}^{r}:=(f)\left\{\begin{array}{l}
\left(\sum_{n=0}^{\infty} a^{n r q}\left(E_{a^{n}}(f)_{p, \lambda}\right)^{q}\right)^{\frac{1}{q}}, 1 \leq q<\infty, \\
\sup _{n \in \mathbb{Z}_{+}} a^{n r} E_{a^{n}}(f)_{p, \lambda}, q=\infty
\end{array}\right.
$$

is finite. In this case the norm (1.21) is equivalent to the norm

$$
\|f\|_{p, \lambda}+\tilde{b}_{p, q, \lambda}^{r}(f) .
$$

The main purpose of this paper is to prove Theorems 1.1 - 1.5. In section 2 we study various properties of Gegenbauer transformation in generalized functions classes, and also obtain some properties of the convolution of functions in these classes. In particular we prove that the Gegenbauer transformation and the convolution defined in the generalized function class are of generalized functions, i.e., they are linear and continuous functions.

The paper is organized as follows:

Some auxiliary results are stared is Section 3 althogh some of them have independent interest. For example, inequality (3.50) is the analogue of classical Nikol'skii-Stechkin inequality. From (3.51), assertion of Theorem 1.3, which is an analogue of classical Bernstein's inequality, follows. Proposition 3.17 and its corollary are analogues of the classical Boas inequality. In section 4 Theorem 1.1 and 1.2 are proved. In section 5 Theorem 1.4 and 1.5 are proved and also obtained various norms of the spaces $B_{p, q, \lambda}^{r}$.

\section{The Gegenbauer Transformations}

The Gegenbauer transformations of the functions $P_{\alpha}^{\lambda}(t)$ and $Q_{\alpha}^{\lambda}(t)$ are defined as the following integral transformations (see [18]):

$$
\begin{aligned}
& F_{P} f(t) \mapsto \hat{f}_{P}(\alpha)=\int_{1}^{\infty} f(t) P_{\alpha}^{\lambda}(t)\left(t^{2}-1\right)^{\lambda-\frac{1}{2}} \mathrm{~d} t \\
& F_{Q} f(t) \mapsto \hat{f}_{Q}(\alpha)=\int_{1}^{\infty} f(t) Q_{\alpha}^{\lambda}(t)\left(t^{2}-1\right)^{\lambda-\frac{1}{2}} \mathrm{~d} t .
\end{aligned}
$$

The inverse Gegenbauer transformations are defined by the following formulas:

$$
\begin{aligned}
& F_{P}^{-1} \hat{f}_{P}(\alpha) \mapsto f(x)=C_{\lambda} \int_{1}^{\infty} \hat{f}_{P}(\alpha) Q_{\alpha}^{\lambda}(x)\left(\alpha^{2}-1\right)^{\lambda-\frac{1}{2}} \mathrm{~d} \alpha, \\
& F_{Q}^{-1} \hat{f}_{Q}(\alpha) \mapsto f(x)=C_{\lambda} \int_{1}^{\infty} \hat{f}_{Q}(\alpha) P_{\alpha}^{\lambda}(x)\left(\alpha^{2}-1\right)^{\lambda-\frac{1}{2}} \mathrm{~d} \alpha,
\end{aligned}
$$

where

$$
C_{\lambda}=\frac{2^{\frac{3}{2}-\lambda} \Gamma\left(\frac{1}{2}\right) \Gamma(\lambda+1) \Gamma\left(\frac{1}{2}-\lambda\right) \Gamma\left(\frac{3}{4}+\frac{\lambda}{2}\right)\left(\Gamma\left(\lambda+\frac{1}{2}\right) \Gamma\left(\frac{5}{4}-\frac{\lambda}{2}\right) \cos \pi \lambda\right)^{-1}}{{ }_{2} F_{1}\left(1, \frac{1}{2}-\lambda ; \frac{5}{4}-\frac{\lambda}{2} ; \frac{1}{2}\right)-{ }_{2} F_{1}\left(1, \frac{1}{2}-\lambda ; \frac{5}{4}-\frac{\lambda}{2} ; \frac{1}{4}-\frac{\lambda}{2}\right)} .
$$

The $\hat{f}_{P}$ transformation (2.1) for any $f \in L_{p, \lambda}(1 \leq p \leq \infty)$ exists that follows from the following inequality (see [18], Lemma 5)

$$
\left|\hat{f}_{P}(\alpha)\right| \lesssim\|f\|_{p, \lambda} \alpha^{2 \lambda-1}
$$

From this it follows that for any $f \in L_{p, \lambda}(1 \leq p \leq \infty) \lim _{n \rightarrow \infty} \hat{f}_{P}(\alpha)=0$. Particulary for $p=1$ we obtain the analogue of Riemann-Lebesgue lemma. On the other hand (see further lemma 3.3), if $f$ and $D_{\lambda}^{k} f$ belong to $L_{p, \lambda}$, then

$$
\widehat{\left(D_{\lambda}^{k} f\right)_{P}}(\alpha)=(\alpha(\alpha+2 \lambda))^{k} \hat{f}_{P}(\alpha) \quad k=1,2, \ldots .
$$

From (2.5) and (2.6) we have 


$$
\left|\hat{f}_{P}(\alpha)\right| \lesssim\left\|D_{\lambda}^{k} f\right\|_{p, \lambda} \alpha^{2 \lambda-1}(\alpha(\alpha+2 \lambda))^{-k}
$$

From this it follows that

$$
\begin{gathered}
\left\|\hat{f}_{P}\right\|_{p, \lambda}^{p}=\int_{1}^{\infty}\left|\hat{f}_{P}(\alpha)\right|^{p}\left(\alpha^{2}-1\right)^{\lambda-\frac{1}{2}} \mathrm{~d} \alpha \\
\lesssim \int_{1}^{\infty} \alpha^{2 \lambda-1}\left(\alpha^{2}-1\right)^{\lambda-\frac{1}{2}} \alpha^{-2 k} \mathrm{~d} \alpha \lesssim \int_{1}^{\infty} \alpha^{\lambda-k-1}(\alpha-1)^{\lambda-\frac{1}{2}} \mathrm{~d} \alpha \\
=\int_{0}^{\infty} \alpha^{\lambda-\frac{1}{2}}(\alpha+1)^{\lambda-k-1} \mathrm{~d} \alpha=\frac{\Gamma\left(\lambda+\frac{1}{2}\right) \Gamma\left(k-2 \lambda+\frac{1}{2}\right)}{\Gamma(k-\lambda+1)}
\end{gathered}
$$

From this and from (2.5) we obtain that $\hat{f}_{P} \in L_{p, \lambda}$ for $1 \leq p \leq \infty$, as

$$
\left\|\hat{f}_{P}\right\|_{\infty, \lambda}=\sup _{1 \leq \alpha<\infty}\left|\hat{f}_{P}(\alpha)\right| \lesssim\|f\|_{p, \lambda} .
$$

Consider the function $\hat{f}_{Q}$ in transformation (2.2). It is clear that for any function in $L_{p, \lambda} \hat{f}_{Q}$ can not exist from the formula (1.3).

Let $S$ be the space of test functions on $[1, \infty)$, that is, $S$ is the set of all infinitely differentiable functions $\varphi(t)$, decaying as $t \rightarrow \infty$, to zero together with all their derivatives, more rapidly than any power of $t^{-1}$. In this way for any test function $\varphi(t)$ the following inequality (see [10], p.7) holds

$$
\left|D^{q} \varphi(t)\right| \leq C_{q r}\left(1+t^{2}\right)^{-r}
$$

with any integer $q \geq 0$ and $r \geq 0$, where the constant $C_{q r}$ does not depend on $t$.

It is clear that the operator $D_{\lambda}$ can apply to test functions infinite times, moreover the estimations

$$
\left|D_{\lambda}^{m} \varphi(t)\right| \lesssim\left(1+t^{2}\right)^{-r}
$$

hold for arbitrary integer $m \geq 0$ and $r \geq 0$.

From (1.4) it follows that

$$
D_{\lambda}^{m} Q_{\alpha}^{\lambda}(x)=(\alpha(\alpha+2 \lambda))^{m} Q_{\alpha}^{\lambda}(x), \alpha \in[1, \infty), m=1,2 \ldots .
$$

From this we have

$$
Q_{\alpha}^{\lambda}(x)=(\alpha(\alpha+2 \lambda))^{-m} D_{\lambda}^{m} Q_{\alpha}^{\lambda}(x) .
$$

In consideration of this equality in $(2.2)$, we obtain

$$
\begin{gathered}
\hat{f}_{Q}(\alpha)=(\alpha(\alpha+2 \lambda))^{-m} \int_{1}^{\infty} f(t) D_{\lambda}^{m} Q_{\alpha}^{\lambda}(t)\left(t^{2}-1\right)^{\lambda-\frac{1}{2}} \mathrm{~d} t \\
=(\alpha(\alpha+2 \lambda))^{-m} \int_{1}^{\infty} Q_{\alpha}^{\lambda}(t) D_{\lambda}^{m} f(t)\left(t^{2}-1\right)^{\lambda-\frac{1}{2}} \mathrm{~d} t,
\end{gathered}
$$

where we use the self-adjointness of the operator $D_{\lambda}$ (see [14], Lemma 4).

Applying inequality (2.8) at $r>\alpha+1$ in (2.9) we have

$$
\left|\hat{f}_{Q}(\alpha)\right| \lesssim(\alpha(\alpha+2 \lambda))^{-m} \int_{1}^{\infty} \frac{Q_{\alpha}^{\lambda}(t)\left(t^{2}-1\right)^{\lambda-\frac{1}{2}}}{\left(1+t^{2}\right)^{\alpha+1}} \mathrm{~d} t .
$$

The Gauss's hypergeometric function ${ }_{2} F_{1}\left(-\frac{\alpha}{2},-\frac{\alpha}{2}+\frac{1}{2} ; 1-\alpha-\lambda ; x^{-2}\right)$ in the formula (1.3) exists by appointed meaning of the parameters for all $x \in[1, \infty)$ (see [11], p.1054). Therefore from (1.3) we have the estimate

$$
Q_{\alpha}^{\lambda}(x)=\frac{\Gamma(2 \lambda) \Gamma(\alpha+1)}{\Gamma(\alpha+2 \lambda)} C_{\alpha}^{\lambda}(x) \lesssim \alpha^{-\lambda}(2 x)^{\alpha} .
$$


Taking into account this inequality in (2.10) we obtain

$$
\begin{gathered}
\left|\hat{f}_{Q}(\alpha)\right| \lesssim \alpha^{-\lambda}(\alpha(\alpha+2 \lambda))^{-m} \int_{1}^{\infty} \frac{\left(t^{2}-1\right)^{\lambda-\frac{1}{2}}(2 t)^{\alpha}}{\left(1+t^{2}\right)^{\alpha+1}} \mathrm{~d} t \\
\lesssim \alpha^{-(2 m+\lambda)} \int_{1}^{\infty} \frac{\left(t^{2}-1\right)^{\lambda-\frac{1}{2}}}{t} \mathrm{~d} t \lesssim \alpha^{-(2 m+\lambda)} \int_{1}^{\infty} t^{-\frac{3}{2}}(t-1)^{\lambda-\frac{1}{2}} \mathrm{~d} t \\
=\alpha^{-(2 m+\lambda)} \int_{0}^{\infty} t^{\lambda-\frac{1}{2}}(t+1)^{\lambda-\frac{3}{2}} \mathrm{~d} t=\frac{\Gamma\left(\lambda+\frac{1}{2}\right) \Gamma(1-\lambda)}{\Gamma\left(\frac{3}{2}\right)} \alpha^{-(2 m+\lambda)} .
\end{gathered}
$$

From this it follows that

$$
\left\|\hat{f}_{Q}\right\|_{\infty, \lambda}=\sup _{1 \leq \alpha<\infty}\left|\hat{f}_{Q}(\alpha)\right| \leq C<\infty .
$$

On the other hand for $1 \leq p<\infty$ we have

$$
\begin{gathered}
\left\|\hat{f}_{Q}\right\|_{p, \lambda}=\left(\int_{1}^{\infty}\left|\hat{f}_{Q}(\alpha)\right|^{p}\left(\alpha^{2}-1\right)^{\lambda-\frac{1}{2}} \mathrm{~d} \alpha\right)^{\frac{1}{p}} \\
\lesssim\left(\int_{1}^{\infty} \frac{\left(\alpha^{2}-1\right)^{\lambda-\frac{1}{2}}}{\alpha^{(2 m+\lambda) p}} \mathrm{~d} \alpha\right)^{\frac{1}{p}} \lesssim\left(\int_{1}^{\infty} \frac{\left(\alpha^{2}-1\right)^{\lambda-\frac{1}{2}}}{\alpha^{2 m+\lambda}} \mathrm{d} \alpha\right)^{\frac{1}{p}} \\
\lesssim\left(\int_{0}^{\infty} \frac{\alpha^{\lambda-\frac{1}{2}}}{(\alpha+1)^{2 m+\lambda}} \mathrm{d} \alpha\right)^{\frac{1}{p}}=\left(\frac{\Gamma\left(\lambda+\frac{1}{2}\right) \Gamma(2 m)}{\Gamma\left(2 m+\lambda+\frac{1}{2}\right)}\right)^{\frac{1}{p}} \leq C<\infty .
\end{gathered}
$$

From this and (2.1) it follows that $\hat{f}_{Q} \in L_{p, \lambda}$ for $f \in S$ and $1 \leq p \leq \infty$.

This shows that the transformations (2.1) and (2.3) also (2.2) and (2.4) are mutual inverse in $S$. According to Theorem 2 in [18], for every function $f$ in $L_{1, \lambda}, F_{P}^{-1} F_{P} f=f$. On the other hand from the symmetry property of the operator $A_{c h t}$ and formula (1.7) we have

$$
\begin{gathered}
f(x)=C_{\lambda} \int_{1}^{\infty} \hat{f}_{P}(\alpha) Q_{\alpha}^{\lambda}(x)\left(\alpha^{2}-1\right)^{\lambda-\frac{1}{2}} \mathrm{~d} \alpha \\
=C_{\lambda} \int_{1}^{\infty}\left(\int_{1}^{\infty} f(t) P_{\alpha}^{\lambda}(t)\left(t^{2}-1\right)^{\lambda-\frac{1}{2}} \mathrm{~d} t\right) Q_{\alpha}^{\lambda}(x)\left(\alpha^{2}-1\right)^{\lambda-\frac{1}{2}} \mathrm{~d} \alpha \\
=\int_{1}^{\infty}\left(C_{\lambda} \int_{1}^{\infty} f(t) Q_{\alpha}^{\lambda}(t)\left(t^{2}-1\right)^{\lambda-\frac{1}{2}} \mathrm{~d} t\right) P_{\alpha}^{\lambda}(t)\left(\alpha^{2}-1\right)^{\lambda-\frac{1}{2}} \mathrm{~d} \alpha \\
=\int_{1}^{\infty} f(\alpha) P_{\alpha}^{\lambda}(x)\left(\alpha^{2}-1\right)^{\lambda-\frac{1}{2}} \mathrm{~d} \alpha,
\end{gathered}
$$

that is, $F_{P} F_{P}^{-1}=f$.

Thus we obtain that

$$
F_{P} F_{P}^{-1} f=f=F_{P}^{-1} F_{P} f
$$

for $f \in L_{1, \lambda}$. Further taking into account (1.7) in (2.3) and (2.4) we obtain that $F_{P}^{-1}=F_{Q}^{-1} \in L_{1, \lambda}$. As in the case of $F_{P}$ we can write that for any $f \in S$

$$
F_{Q} F_{Q}^{-1} f=f=F_{Q}^{-1} F_{Q} f
$$

and

$$
F_{P}^{-1} F_{P} f=f=F_{Q}^{-1} F_{Q} f .
$$

Since $S \subset L_{1, \lambda}$, from above results we confine that (2.1) and (2.3) and also (2.2) and (2.4) are mutual inverse in $S$. 
Let $S^{\prime}$ be the set of continuous linear functionals on $S$, that is, $S^{\prime}$ is the space of temperate generalized functions. For $f \in S^{\prime}$ and $\varphi \in S$ we denote by $f(\varphi)=\langle f, \varphi\rangle$ the value of the functional $f$ at $\varphi$. We denote the spaces $L_{p, \lambda}, 1 \leq p \leq \infty$, in $S^{\prime}$ by putting

$$
<f, \varphi>:=\int_{1}^{\infty} f(t) \varphi(t)\left(t^{2}-1\right)^{\lambda-\frac{1}{2}} \mathrm{~d} t
$$

for $f \in L_{p, \lambda}$ and $\varphi \in S$.

The following Parseval equality holds for the Gegenbauer transformation (see [18], Lemma 8)

$$
\int_{1}^{\infty} f(x) g(x)\left(x^{2}-1\right)^{\lambda-\frac{1}{2}} \mathrm{~d} x=C_{\lambda} \int_{1}^{\infty} \hat{f}_{P}(\alpha) \hat{g}_{Q}(\alpha)\left(\alpha^{2}-1\right)^{\lambda-\frac{1}{2}} \mathrm{~d} \alpha
$$

for $f, g \in S$.

From this it follows that if $f \in S$, then take place the equality:

$$
\int_{1}^{\infty} f^{2}(x)\left(x^{2}-1\right)^{\lambda-\frac{1}{2}} \mathrm{~d} x=C_{\lambda} \int_{1}^{\infty} \hat{f}_{P}(\alpha) \hat{f}_{Q}(\alpha)\left(\alpha^{2}-1\right)^{\lambda-\frac{1}{2}} \mathrm{~d} \alpha,
$$

which we remind of the Parseval-Plancherel's formula.

Equality (2.14) can be written as

$$
<f, g>=C_{\lambda}<F_{P} f, F_{Q} g>\text {. }
$$

Taking into account (2.5), we transform last equality to form

$$
<F_{P} f, g>=\frac{1}{C_{\lambda}}<f, F_{Q}^{-1} g>=<f, \stackrel{\vee}{g}_{Q}>,
$$

where $\stackrel{\vee}{g}_{Q}=\frac{1}{C_{\lambda}} F_{Q}^{-1} g$ which follows from equality (2.12).

The Gegenbauer transformation can be extended to a class of functions wider than $S$.

We show that the Gegenbauer transformation $F_{P} f$ of generalized functions next are the generalized functions, i.e. of continuous linear functionals, which defined by properties:

1) $<F_{P} f, \varphi+\psi>=<F_{P} f, \varphi>+<F_{P} f \psi>, \varphi, \psi \in S$,

2) $<F_{P} f, \alpha \varphi>=\alpha<F_{P} f, \varphi>, \alpha \in \mathbb{R}$,

3) if $\varphi_{n} \rightarrow 0$, then $<F_{P} f, \varphi_{n}>\rightarrow 0$ at $n \rightarrow \infty$. $3)$.

First two properties follow from the linearity property of the integrals. We will only prove the property

The convergence to zero of the sequence $\varphi_{n} \in S$ is defined by the condition: for any natural numbers $r$ and $q$ and positive $\varepsilon$ there exists a natural number such that whenever $n \geq n_{0}$ the inequality

$$
\left(1+x^{2}\right)^{r}\left|\varphi_{n}^{(q)}(x)\right|<\varepsilon
$$

holds (see [10]).

Then we have

$$
\begin{gathered}
<F_{P} f, \varphi_{n}>=\int_{1}^{\infty} F_{P} f(\alpha) \varphi_{n}(\alpha)\left(\alpha^{2}-1\right)^{\lambda-\frac{1}{2}} \mathrm{~d} \alpha \\
=\int_{1}^{A} F_{P} f(\alpha) \varphi_{n}(\alpha)\left(\alpha^{2}-1\right)^{\lambda-\frac{1}{2}} \mathrm{~d} \alpha \\
+\int_{A}^{\infty} F_{P} f(\alpha) \varphi_{n}(\alpha)\left(\alpha^{2}-1\right)^{\lambda-\frac{1}{2}} \mathrm{~d} \alpha=J_{1}+J_{2} .
\end{gathered}
$$

Since (see [18]) $F_{P} f(\alpha) \rightarrow 0$ as $\alpha \rightarrow \infty$, we choose a number $A$ so that for given $\varepsilon>0$ the inequality

$$
\left|F_{P} f(\alpha)\right|<\varepsilon \text { for } \alpha>A
$$

holds. 
Then

$$
\left[J_{2}\right]<\varepsilon \int_{A}^{\infty}\left|\varphi_{n}(\alpha)\right|\left(\alpha^{2}-1\right)^{\lambda-\frac{1}{2}} \mathrm{~d} \alpha .
$$

As $\varphi_{n} \in S$, then by definition we have

$$
\left|\varphi_{n}(\alpha)\right| \leq \frac{c_{r}}{\left(1+\alpha^{2}\right)^{r}}, r=1,2, \ldots
$$

Taking into account (2.20) in (2.19) we obtain

$$
\begin{aligned}
\left|J_{2}\right| & <c_{r} \varepsilon \int_{A}^{\infty} \frac{\left(\alpha^{2}-1\right)^{\lambda-\frac{1}{2}} \mathrm{~d} \alpha}{\left(1+\alpha^{2}\right)^{r}}=c_{r} \varepsilon \int_{A}^{\infty} \frac{(\alpha-1)^{\lambda-\frac{1}{2}} \mathrm{~d} \alpha}{(\alpha+1)^{\frac{1}{2}-\lambda}\left(1+\alpha^{2}\right)^{r}} \\
& \leq c_{r} \varepsilon \int_{0}^{\infty} \frac{(\alpha-1)^{\lambda-\frac{1}{2}} \mathrm{~d} \alpha}{\alpha^{2 r+\frac{1}{2}-\lambda}}=c_{r} B\left(\lambda+\frac{1}{2}, 2 r-2 \lambda\right) \varepsilon,
\end{aligned}
$$

where $B(a, b)$ is of Euler integrals first type.

Since $\varphi_{n}(\alpha) \rightarrow 0$ as $n \rightarrow \infty$, by (2.17) we have

$$
\left|\varphi_{n}(\alpha)\right| \leq \frac{\varepsilon}{\left(1+\alpha^{2}\right)^{r}}, r=1,2, \ldots .
$$

From here we have

$$
\left|J_{1}\right| \leq \varepsilon \int_{1}^{A} \frac{\left|F_{P} f(\alpha)\right|\left(\alpha^{2}-1\right)^{\lambda-\frac{1}{2}}}{\left(1+\alpha^{2}\right)^{r}} \mathrm{~d} \alpha .
$$

Take into account (2.5), we obtain

$$
\begin{aligned}
\left|J_{1}\right| & \leq \varepsilon \int_{1}^{A} \frac{(\alpha-1)^{\lambda-\frac{1}{2}} \mathrm{~d} \alpha}{\left(1+\alpha^{2}\right)^{r} \alpha^{1-2 \lambda}} \leq \varepsilon c_{r} \int_{1}^{A}(\alpha-1)^{\lambda-\frac{1}{2}} \mathrm{~d} \lambda \\
& =\left.c(\lambda, r) \varepsilon(\alpha-1)^{\lambda+\frac{1}{2}}\right|_{1} ^{A}=c(\lambda, r)(A-1)^{\lambda+\frac{1}{2}} \varepsilon .
\end{aligned}
$$

Taking into account (2.21) and (2.22) in (2.18), we obtain

$$
<F_{P} f, \varphi_{n}>\rightarrow 0 \text { as } n \rightarrow \infty \text {. }
$$

In this way, the Gegenbauer transformation $F_{P} f$ for $f \in S^{\prime}$ next is the generalized function, i.e. $F_{P} f \in S^{\prime}$. Therefore the formula $(2.16)$ can be naturally extended on functions from $S^{\prime}$, i.e., for $f \in S^{\prime}$ by definition

$$
<F_{P} f, g>:=\frac{1}{C_{\lambda}}<f, F_{P}^{-1} g>, \quad g \in S .
$$

We note an important property of Gegenbauer transformation (see [14], Lemma 3.3): if $f \in S^{\prime}$, then the following inequality holds:

$$
\left(\widehat{D_{\lambda}^{k}} f\right)_{P}(\alpha)=(\alpha(\alpha+2 \lambda))^{k} \hat{f}_{P}(\alpha), \quad k=1,2 \ldots
$$

In the class $S$, the operator $D_{\lambda}$ is symmetric in the following sence (see [14], Lemma 4), i.e.,

$$
<D_{\lambda} \psi, \varphi>=<\psi, D_{\lambda} \varphi>, \quad \varphi, \psi \in S,
$$

that is equivalent

$$
\int_{0}^{\infty}\left(D_{\lambda} \psi\right)(\operatorname{ch} x) \varphi(\operatorname{ch} x) \operatorname{sh}^{2 \lambda} x \mathrm{~d} x=\int_{0}^{\infty} \psi(\operatorname{ch} x)\left(D_{\lambda} \varphi\right)(\operatorname{ch} x) \operatorname{sh}^{2 \lambda} x \mathrm{~d} x .
$$

Reflections

$$
\varphi \mapsto \int_{0}^{\infty}\left(D_{\lambda} \psi\right)(\operatorname{ch} x) \varphi(\operatorname{ch} x) \operatorname{sh}^{2 \lambda} x \mathrm{~d} x
$$


and

$$
f \mapsto \int_{0}^{\infty} \psi(\operatorname{ch} x) f(\operatorname{ch} x) \operatorname{sh}^{2 \lambda} x \mathrm{~d} x
$$

define the continuous linear functionals on $S$. Denoting their over $D_{\lambda} \psi$ and $\psi$, we rewrite the last equality by form

$$
\left(D_{\lambda} \psi\right)(\varphi)=\psi\left(D_{\lambda} \varphi\right)
$$

or the same

$$
<D_{\lambda} \psi, \varphi>=<\psi, D_{\lambda}, \varphi>, \quad \psi \in S^{\prime}, \quad \varphi \in S .
$$

Since, right-hand side of (2.25) is defined for all $\psi \in S^{\prime}$ and $\varphi \in S$, and representation $\varphi \rightarrow \psi\left(D_{\lambda} \varphi\right)$ is continuous on $S$, as superposition of two continuous functions, then we can define $D_{\lambda} \psi$ of generalized function $\psi \in S^{\prime}$ by means of $(2.25)$.

Obviously, that then $D_{\lambda} \psi \in S^{\prime}$.

From inequalities (1.10), (2.5) and also of the self-adjoind of the operator $A_{c h t}^{\lambda}$ follows that for $\varphi \in L_{1, \lambda}$ the equality holds:

$$
<A_{c h t}^{\lambda} \hat{f}_{P}, \varphi>=<\hat{f}_{P}, A_{c h t}^{\lambda} \varphi>.
$$

Since $\hat{f}_{P}(\alpha) \in S^{\prime}$, then naturally extended the generalized Gegenbauer shift operator on generalized functions space by formula

$$
<A_{c h t}^{\lambda} f, \varphi>:=<f, A_{c h t}^{\lambda} \varphi>, f \in S^{\prime}, \varphi \in S .
$$

Let $a$ be arbitrary positive number. The dilation operator (see [16])

$$
f_{a}(\operatorname{ch} x)=\frac{f\left(\operatorname{ch} \frac{x}{a}\right) \operatorname{sh}^{2 \lambda} \frac{x}{a}}{a \operatorname{sh}^{2 \lambda} x}, a>0
$$

of the function in $L_{1, \lambda}$ preserve meaning of the integral, that is

$$
\int_{0}^{\infty} f_{a}(\operatorname{ch} x) \operatorname{sh}^{2 \lambda} x \mathrm{~d} x=\int_{0}^{\infty} f(\operatorname{ch} x) \operatorname{sh}^{2 \lambda} x \mathrm{~d} x .
$$

Really,

$$
\begin{gathered}
\int_{0}^{\infty} f_{a}(\operatorname{ch} x) \operatorname{sh}^{2 \lambda} x \mathrm{~d} x=\int_{0}^{\infty} \frac{f\left(\operatorname{ch} \frac{x}{a}\right) \operatorname{sh}^{2 \lambda} \frac{x}{a}}{a \operatorname{sh}^{2 \lambda} x} \operatorname{sh}^{2 \lambda} x \mathrm{~d} x \\
=\int_{0}^{\infty} f\left(\operatorname{ch} \frac{x}{a}\right) \operatorname{sh}^{2 \lambda} \frac{x}{a} d \frac{x}{a}=\int_{0}^{\infty} f(\operatorname{ch} x) \operatorname{sh}^{2 \lambda} x \mathrm{~d} x .
\end{gathered}
$$

From (2.29) it follows that

$$
\left\|f_{a}\right\|_{1, \lambda}=\|f\|_{1, \lambda} .
$$

The following correlations is connect of the generalized Gegenbauer shift operator and of Gegenbauer transformation (see [18], Lemma 1):

$$
\left(\widehat{A_{c h t} f}\right)_{P}(\alpha)=\hat{f}_{P}(\alpha) Q_{\alpha}^{\lambda}(\operatorname{cht}), \quad \alpha \in[1, \infty), \quad t \in[0, \infty) .
$$

The operators $A_{c h t}$ and $D_{\lambda}$ commute, that is,

$$
\left(D_{\lambda} A_{\text {cht }} f\right)(\operatorname{ch} x)=\left(A_{\text {ch } t} D_{\lambda} f\right)(\operatorname{ch} x), \quad f \in S .
$$

This equality is a particular case of more qeneral equality (see further Lemma 3.4)

$$
\left(A_{\text {ch } t}^{k} D_{\lambda}^{r} f\right)(\operatorname{ch} x)=\left(D_{\lambda}^{r} A_{c h t} f\right)(\operatorname{ch} x), \quad k, r=1,2, \ldots, \quad f \in S,
$$

although it can be proved by application of the Gegenbauer transformation of both side of (2.32). Taking into account (2.6) and (2.30), we have

$$
\left(\widehat{D_{\lambda} A_{c h t} f}\right)_{P}(\alpha)=\alpha(\alpha+2 \lambda)\left(\widehat{A_{c h t} f}\right)_{P}(\alpha)=\alpha(\alpha+2 \lambda) Q_{\alpha}^{\lambda}(\operatorname{cht}) \hat{f}_{P}(\alpha)
$$




$$
=\hat{f}_{P}(\alpha) D_{\lambda} Q_{\alpha}^{\lambda}(\operatorname{cht})=\left(\widehat{A_{\operatorname{cht} D_{\lambda}} f}\right)_{P}(\alpha)
$$

from this (2.32) follows.

The convolution of functions $f$ and $g$ on $\mathbb{R}_{+}$is defined by the relation

$$
(f * g)(\operatorname{ch} x)=\int_{0}^{\infty} g(\operatorname{cht} t) A_{\operatorname{ch} t} f(\operatorname{ch} x) \operatorname{sh}^{2 \lambda} t \mathrm{~d} t .
$$

The convolution has sense if the integral on the right-hand side of (2.28) is convergence. If $f \in L_{p, \lambda}$, $g \in L_{1, \lambda}$, then the convolution is defined and belong $L_{p, \lambda}, 1 \leq p \leq \infty$, as (see [18], Lemma 4)

$$
\|f * g\|_{p, \lambda} \leq\|f\|_{p, \lambda} \cdot\|g\|_{1, \lambda} .
$$

From here, in particular it follows that for $f, g \in S$ their convolution $f * g \in S$.

We deriv some properties of the convolution.

1. The convolution is commutative, that is,

$$
f * g=g * f .
$$

2.

$$
(\widehat{f * g})_{P}(\alpha)=\hat{f}_{P}(\alpha) \hat{g}_{Q}(\alpha),
$$

from this and also (2.12) it follows that 3.

$$
f * g=F_{P}^{-1}\left(\hat{f}_{P} \hat{g}_{Q}\right) .
$$

4.

$$
D_{\lambda}^{r}(f * g)=\left(D_{\lambda}^{r} f\right) * g=f *\left(D_{\lambda}^{r} g\right), \quad r=1,2 \ldots,
$$

which follows from (2.36) by using (2.6).

Indeed,

$$
\begin{gathered}
\left(\widehat{D_{\lambda}^{r f} * g}\right)_{P}(\alpha)=\left(\widehat{D_{\lambda}^{r} f}\right)_{P}(\alpha) \hat{g}_{Q}(\alpha)=(\alpha(\alpha+2 \lambda)) \hat{f}_{P}(\alpha) \hat{g}_{Q}(\alpha) \\
=(\alpha(\alpha+2 \lambda))^{r}(\widehat{f * g})_{P}(\alpha)=\left(\widehat{D_{\lambda}^{r}(f * g)}\right)_{P}(\alpha) .
\end{gathered}
$$

From this and (2.35) it follows that (2.38).

5. It is associative, that is,

$$
(f * g) * h=f *(g * h) .
$$

Indeed, by using commutativity of the operator $A_{c h}$, we obtain

$$
\begin{aligned}
& ((f * g) * g)(\operatorname{ch} x)=\int_{0}^{\infty}(f * g)(\operatorname{cht} t) A_{\text {ch } t} h(\operatorname{ch} x) \operatorname{sh}^{2 \lambda} t \mathrm{~d} t \\
& =\int_{0}^{\infty}\left(\int_{0}^{\infty} f(\operatorname{ch} x) A_{\operatorname{ch} x} g(\operatorname{cht}) \operatorname{sh}^{2 \lambda} x \mathrm{~d} x\right) A_{\operatorname{ch} t} h(\operatorname{ch} x) \operatorname{sh}^{2 \lambda} t \mathrm{~d} t \\
& =\int_{0}^{\infty} \int_{0}^{\infty} g(\operatorname{ch} x) A_{\operatorname{ch} x} f(\operatorname{cht} t) A_{\operatorname{ch} t} h(\operatorname{ch} x) \operatorname{sh}^{2 \lambda} x \operatorname{sh}^{2 \lambda} t \mathrm{~d} x \mathrm{~d} t \\
& =\int_{0}^{\infty} \int_{0}^{\infty} g(\operatorname{ch} x) A_{\operatorname{cht}} f(\operatorname{ch} x) A_{\operatorname{ch} x} h(\operatorname{cht} t) \operatorname{sh}^{2 \lambda} x \operatorname{sh}^{2 \lambda} t \mathrm{~d} x \mathrm{~d} t \\
& =\int_{0}^{\infty}(g * h)(\operatorname{cht} t) A_{c h t} f(\operatorname{ch} x) \operatorname{sh}^{2 \lambda} t \mathrm{~d} t=(f *(g * h))(\operatorname{ch} x) .
\end{aligned}
$$

These properties hold for $f, g, h \in S$.

In case then $f \in S^{\prime}$ and $g \in S$ the convolution $f * g$ is defined by formula

$$
(f * g)(\operatorname{ch} x):=<f, A_{\operatorname{ch} x} g>=f\left(A_{\operatorname{ch} x} g\right), \quad x \in \mathbb{R}_{+} .
$$


The right-hand of (2.39) exist, so far as $f$ is slow growing function, and $A_{c h}{ }_{x} g \in S$ on the strength inequality (1.10). Moreover the reflection $g \mapsto f\left(A_{c h} g\right)$ is continuous as superposition two continuous functions.

Theorem 2.1. Let $f \in S^{\prime}$ and $g \in S$. Then their convolution $u($ ch $x)=f\left(A_{\text {ch }}{ }_{x} g\right)$ are generalized function slow growing, i.e. $u \in S^{\prime}$.

Proof. First we show, that $u \in C^{\infty}$. Using (2.33), we can write

$$
D_{\lambda}^{k} u(\operatorname{ch} x)=\int_{0}^{\infty} f(\operatorname{ch} t) A_{\operatorname{ch} x} D_{\lambda}^{k} g(\operatorname{ch} t) \operatorname{sh}^{2 \lambda} t \mathrm{~d} t=f\left(A_{\operatorname{ch} x} D_{\lambda}^{k} g\right), k=1,2, \ldots
$$

As, $g \in S$, then $D_{\lambda}^{k} g$ exists and continuity for any $k \in \mathbb{N}$. But then on the strength of continuous of the operator $A_{c h x}^{\lambda}$ is continuous and the right-hand side (2.40), as superposition two continuous functions. In this way $u \in C^{\infty}$.

We show that $u$ is slow growing function. Taking into account of Taylor-Delsart formula (see [14], Lemma 6), and also of Lemma 3.6 (see further), we obtain

$$
A_{c h t}^{\lambda} f(\operatorname{ch} x)=\sum_{\nu=0}^{n-1} C_{\nu}(\operatorname{cht} t) D_{\lambda}^{\nu} f(\operatorname{ch} x)+C_{n}(\operatorname{cht}) A_{c h t}^{\lambda} D_{\lambda}^{n} f(\operatorname{ch} x) .
$$

From here and from (2.39) we have

$$
\begin{aligned}
& u(\operatorname{ch} x)=\int_{0}^{\infty} g(\operatorname{cht}) A_{c h t}^{\lambda} f(\operatorname{ch} x) s h^{2 \lambda} t \mathrm{~d} t \\
= & \sum_{\nu=0}^{n-1} D_{\lambda}^{\nu} f(\operatorname{ch} x) \int_{0}^{\infty} C_{\nu}(\operatorname{cht}) g(\operatorname{ch} t) \operatorname{sh}^{2 \lambda} t \mathrm{~d} t \\
+ & \int_{0}^{\infty} C_{n}(\operatorname{cht} t) A_{\text {ch } t} D^{n} f(\operatorname{ch} x) g(\operatorname{cht} t) s h^{2 \lambda} t \mathrm{~d} t .
\end{aligned}
$$

Since

$$
\operatorname{ch}(x-t) \leq \operatorname{ch} x \operatorname{ch} t-\operatorname{sh} x \operatorname{sh} t \cos \varphi \leq \operatorname{ch}(x+t),
$$

then

$$
\left|A_{c h t}^{\lambda} f(\operatorname{ch} x)\right| \leq \sup _{1 \leq u \leq c h(x+t)}|f(u)| .
$$

But then

$$
\begin{aligned}
& \left|\int_{0}^{\infty} C_{n}(\operatorname{ch} t) A_{\text {ch } t} D_{\lambda}^{n} f(\operatorname{ch} x) g(\operatorname{ch} t) \operatorname{sh}^{2 \lambda} t \mathrm{~d} t\right| \\
\leq & \sup _{1 \leq u \leq c h(x+t)}\left|D_{\lambda}^{n} f(u)\right| \int_{0}^{\infty} C_{k}(\operatorname{ch} t)|g(\operatorname{ch} t)| \operatorname{sh}^{2 \lambda} t \mathrm{~d} t .
\end{aligned}
$$

Further, since (see [14], Lemma 5)

$$
C_{k}(\operatorname{ch} t) \leq c(k, \lambda)(\operatorname{ch} t-1)^{k}, k=1,2, \ldots,
$$

but $g \in S$, that the integral

$$
\int_{0}^{\infty} C_{k}(\operatorname{ch} t)|g(\operatorname{ch} t)| \operatorname{sh}^{2 \lambda} t \mathrm{~d} t
$$

exist. But then from (2.42) and (2.41) it follows that

$$
u(\operatorname{ch} x) \lesssim \sum_{\nu=0}^{n} \sup _{1 \leq u \leq c h(x+t)} D_{\lambda}^{\nu} f(u)
$$


Since (see [14], Lemma 1)

$$
D_{\lambda} f(x)=\left(x^{2}-1\right) f^{\prime \prime}(x)+(2 \lambda+1) x f^{\prime}(x),
$$

it follows that the convolution $u(\operatorname{ch} x)$ has growth polynomials from (2.43), i.e., it is a slow growing function.

Thus the theorem is proved.

Now let $f, g, h \in S$. Using the Fubini theorem we show that

$$
\begin{gathered}
(f * g) *(h)=<f * g, h> \\
=\int_{0}^{\infty}\left(\int_{0}^{\infty} A_{c h t}^{\lambda} f(\operatorname{ch} x) g(\operatorname{cht}) s h^{2 \lambda} t \mathrm{~d} t\right) h(\operatorname{ch} x) s h^{2 \lambda} x \mathrm{~d} x \\
=\int_{0}^{\infty}\left(\int_{0}^{\infty} A_{c h t}^{\lambda} f(\operatorname{ch} x) h(\operatorname{ch} x) s h^{2 \lambda} x \mathrm{~d} x\right) g(\operatorname{ch} t) s h^{2 \lambda} t \mathrm{~d} t \\
=\int_{0}^{\infty}\left(\int_{0}^{\infty} f(\operatorname{ch} x) A_{c h t}^{\lambda} h(\operatorname{ch} x) s h^{2 \lambda} x \mathrm{~d} x\right) g(\operatorname{cht} t) s h^{2 \lambda} t \mathrm{~d} t \\
=<g, f * h>=<g * f, h>=<f, g * h>,
\end{gathered}
$$

i.e.,

$$
(f * g) *(h)=f *(g * h) .
$$

If $f \in S^{\prime}$ and $g, h \in S$, then right-hand side of the last equality is defined since $g * h \in S$. Moreover, the representation $h \mapsto f(g * h)$ is continuous which is the superposition of two functions. In this way the convolution of the generalized function $f$ and a basic function $g$ can be defined by the following equality

$$
<f * g, h>=<f, g * h>, f \in S^{\prime}, \quad g, h \in S .
$$

We show that for all $f \in S^{\prime}$ and $g, h \in S$ the convolution is associative, that is,

$$
(f * g) * h=f *(g * h) .
$$

Indeed, if $f \in S^{\prime}$, and $g, h \in S$, then

$$
\begin{aligned}
& (f * g) * h(\operatorname{ch} x)=\int_{0}^{\infty}(f * g)(\operatorname{cht}) A_{c h t}^{\lambda} h(\operatorname{ch} x) s h^{2 \lambda} t \mathrm{~d} t \\
= & \int_{0}^{\infty}\left(\int_{0}^{\infty} f(\operatorname{ch} x) A_{c h x}^{\lambda} g(\operatorname{cht}) \operatorname{sh}^{2 \lambda} x \mathrm{~d} x\right) A_{c h t}^{\lambda} h(\operatorname{ch} x) \operatorname{sh}^{2 \lambda} t \mathrm{~d} t \\
= & \int_{0}^{\infty} \int_{0}^{\infty} g(\operatorname{ch} x) A_{c h x}^{\lambda} f(\operatorname{cht}) A_{c h x}^{\lambda}(\operatorname{ch} x) s h^{2 \lambda} x s h^{2 \lambda} t \mathrm{~d} x \mathrm{~d} t \\
= & \int_{0}^{\infty} \int_{0}^{\infty} g(\operatorname{ch} x) A_{c h x}^{\lambda} f(\operatorname{ch} x) A_{c h x}^{\lambda}(\operatorname{cht}) \operatorname{sh}^{2 \lambda} x \operatorname{sh}^{2 \lambda} t \mathrm{~d} x \mathrm{~d} t \\
= & \int_{0}^{\infty}(g * h)(\operatorname{cht}) A_{c h t}^{\lambda} f(\operatorname{ch} x) \operatorname{sh}^{2 \lambda} t \mathrm{~d} t=f *(g * h)(\operatorname{ch} x) .
\end{aligned}
$$

Since $g, h \in S$, that their convolution $g * h$ belongs to $S$, but the right-hand part of (2.45) according to (2.44) it is convolution of the shift function $f \in S^{\prime}$ and the test function $g * h \in S$.

We show that the convolution of $f \in S^{\prime}$ and $g \in S$ is defined by formula (2.39). It remains to show that the properties (2.35), (2.36) and (2.38) are provided.

Indeed, using (2.26) and (2.39) we obtain

$$
(f * g)(\operatorname{ch} x)=<f, A_{\text {ch }{ }_{t}}^{\lambda} g>=<A_{\text {ch } t}^{\lambda} f, g>=(g * f)(\operatorname{ch} x) .
$$


Further, using equalities (2.46), and also (1.7) and by changing the order of integration we obtain

$$
\begin{gathered}
(\widehat{f * g})_{P}(\alpha)=\int_{0}^{\infty}(f * g)(\operatorname{ch} x) P_{\alpha}^{\lambda}(\operatorname{ch} x) \operatorname{sh}^{2 \lambda} x \mathrm{~d} x \\
=\int_{0}^{\infty}\left(\int_{0}^{\infty} g(\operatorname{cht} t) A_{\text {ch } t} f(\operatorname{ch} x) s h^{2 \lambda} t \mathrm{~d} t\right) P_{a}^{\lambda}(\operatorname{ch} x) \operatorname{sh}^{2 \lambda} x \mathrm{~d} x \\
=\int_{0}^{\infty}\left(\int_{0}^{\infty} f(\operatorname{cht} t) A_{\operatorname{ch} t} P_{a}^{\lambda}(\operatorname{ch} x) \operatorname{sh}^{2 \lambda} t \mathrm{~d} t\right) g(\operatorname{ch} x) \operatorname{sh}^{2 \lambda} x \mathrm{~d} x \\
=\int_{0}^{\infty}\left(\int_{0}^{\infty} f(\operatorname{cht} t) P_{\alpha}^{\lambda}(\operatorname{cht} t) \operatorname{sh}^{2 \lambda} t \mathrm{~d} t\right) g(\operatorname{ch} x) Q_{\alpha}^{\lambda}(\operatorname{ch} x) \operatorname{sh}^{2 \lambda} x \mathrm{~d} x=\hat{f}_{P}(\alpha) \hat{g}_{Q}(\alpha),
\end{gathered}
$$

i.e., take place (2.36).

Using consistent of the formulas (2.40), (2.39), (2.25), (2.32) and (2.26), we obtain

$$
\begin{gathered}
D_{\lambda}^{r}(f * g)=f\left(A_{\text {ch } x} D_{\lambda}^{r} g\right)=<f, A_{\text {ch } x} D_{\lambda}^{r} g>=f *\left(D_{\lambda}^{r} g\right)=<A_{\text {ch } x} f, D_{\lambda}^{r} g> \\
=<D_{\lambda}^{r} A_{\text {ch } x} f, g>=<A_{\text {ch } x} D_{\lambda}^{r} f, g>=<D_{\lambda}^{r} f, A_{\text {ch } x} g>=\left(D_{\lambda}^{r} f\right) * g,
\end{gathered}
$$

i.e., take place (2.38).

\section{Auxiliary Results}

For the proof of Theorems 1.1 and 1.2 we need some auxiliary assertions.

We consider the function

$$
C_{k}(\operatorname{ch} x)=-\int_{1}^{c h x} \theta(\operatorname{ch} x, \sigma) C_{k-1}(\sigma)\left(\sigma^{2}-1\right)^{\lambda-\frac{1}{2}} d \sigma, C_{0}=1, k=1,2, \ldots,
$$

where

$$
\begin{aligned}
\theta(\operatorname{ch} x, \sigma) & = \begin{cases}-\int_{\sigma}^{c h x}\left(u^{2}-1\right)^{-\lambda-\frac{1}{2}} d u, & 1<\sigma<\operatorname{ch} x, \\
0, & \text { for the other } \sigma,\end{cases} \\
R_{1}(\operatorname{chs}) f(\operatorname{ch} x) & =\int_{1}^{c h s} \theta(\operatorname{chs}, \sigma)\left(A_{\sigma} D_{\lambda} f\right)(\operatorname{ch} x)\left(\sigma^{2}-1\right)^{\lambda-\frac{1}{2}} d \sigma,
\end{aligned}
$$

and

$$
\begin{gathered}
R_{k}(\operatorname{chs}) f(\operatorname{ch} x)=\int_{1}^{c h s} \theta(\operatorname{chs}, \sigma)\left(R_{k-1}(\sigma) D_{\lambda} f\right)(\operatorname{ch} x)\left(\sigma^{2}-1\right)^{\lambda-\frac{1}{2}} d \sigma \\
k=2,3, \ldots
\end{gathered}
$$

The following assertion is just (see [13]):

Lemma 3.1. [14] If $f \in D_{\lambda}^{n-1}[0, \infty)$, then the Taylor-Delsarte's formula is valid:

$$
\begin{gathered}
R_{n}(\operatorname{chs}) f(\operatorname{ch} x)=A_{\text {cht }} f(\operatorname{ch} x)-\sum_{k=0}^{n-1} C_{k}\left(\operatorname{chs} s D_{\lambda}^{k} f(\operatorname{ch} x), n=1,2, \ldots,\right. \\
D_{\lambda}^{0} f(\operatorname{ch} x) \equiv f(\operatorname{ch} x) .
\end{gathered}
$$

Lemma 3.2. Let $f \in L_{p, \lambda}$. Then the following equality is valid:

$$
\left(\widehat{A_{\text {cht }}^{k} f}\right)_{P}(\alpha)=\widehat{f}_{P}(\alpha)\left(Q_{\alpha}^{\lambda}(\text { cht })\right)^{k}, \quad k=1,2, \ldots .
$$


Lemma 3.3. Let $f \in W_{p, \lambda}^{k}$, then

$$
\left(\widehat{D_{\lambda}^{k} f}\right)_{P}(\alpha)=(\alpha(\alpha+2 \lambda))^{k} \widehat{f}_{P}(\alpha), \quad k=1,2, \ldots .
$$

Lemma 3.4. If $f \in S$, then following equality is valid:

$$
\left(A_{c h t}^{k} D_{\lambda}^{r} f\right)(\operatorname{ch} x)=\left(D_{\lambda}^{r} A_{c h t}^{k} f\right)(\operatorname{ch} x), \quad k, r=1,2, \ldots .
$$

Lemma 3.5. Following equality is valid:

$$
A_{\text {cht }}^{k} R_{n}(\operatorname{chs}) f(\operatorname{ch} x)=R_{n}(\operatorname{chs})\left(A_{\text {chs }}^{k} f\right)(\operatorname{ch} x), \quad k=1,2, \ldots .
$$

Lemma 3.6. The following equality is valid:

$$
R_{n}(\operatorname{chs}) f(\operatorname{ch} x)=C_{n}(\operatorname{chs}) A_{\text {chs }} D_{\lambda}^{n} f(\operatorname{ch} x), \quad n=1,2, \ldots .
$$

Lemma 3.7. If $f \in D_{\lambda}^{k}[0, \infty)$, then the following equality is valid:

$$
\Delta_{\text {chs }}^{k} f(\operatorname{ch} x)=R_{k}(\operatorname{chs})\left(A_{\text {chs }}^{k-1} f\right)(\operatorname{ch} x), k=1,2, \ldots .
$$

Lemma 3.8. If the function $f \in D_{\lambda}^{k}[0, \infty)$, then the following equality is valid:

$$
\Delta_{\text {chs }}^{k} f(\operatorname{ch} x)=C_{k}(\operatorname{chs}) A_{c h s}^{k}\left(D_{\lambda}^{k} f\right)(\operatorname{ch} x), k=1,2, \ldots .
$$

Lemma 3.9. If $f \in D_{\lambda}^{k}[0, \infty)$, then the following equality is valid:

$$
\Delta_{\text {cht }}^{m} D_{\lambda}^{k} f(\operatorname{ch} x)=D_{\lambda}^{k} \Delta_{c h t}^{m} f(\operatorname{ch} x), k=1,2, \ldots ; m=1,2, \ldots .
$$

Lemma 3.10. For any function $f \in W_{p, \lambda}^{k}, k=1,2, \ldots, 1 \leq p \leq \infty$ the following inequality is valid:

$$
\left\|\Delta_{c h t}^{k} f\right\|_{p, \lambda} \leq \frac{\Gamma(2 \lambda+1)}{\Gamma(k+1) \Gamma(2 k+2 \lambda-1)}(\operatorname{ch} t-1)^{k}\left\|D_{\lambda}^{k} f\right\|_{p, \lambda} .
$$

Proof. Since $f \in W_{p, \lambda}^{k}$, then from of the equality (3.2) it follows that

$$
\left\|\Delta_{c h t}^{k} f\right\|_{p, \lambda} \leq C_{k}(\operatorname{cht})\left\|\left(A_{c h t}^{k} D_{\lambda}^{k}\right) f\right\|_{p, \lambda} .
$$

Taking into account (1.10) and also the inequality (see [14], Lemma 5)

$$
C_{k}(\operatorname{ch} t) \leq \frac{\Gamma(2 \lambda+1)}{\Gamma(k+1) \Gamma(2 k+2 \lambda-1)}(\operatorname{ch} t-1)^{k},
$$

we obtain

$$
\left\|\Delta_{c h t}^{k} f\right\|_{p, \lambda} \leq C_{k}(\operatorname{ch} t)\left\|D_{\lambda}^{k} f\right\|_{p, \lambda} \leq \frac{\Gamma(2 \lambda+1)}{\Gamma(k+1) \Gamma(2 k+2 \lambda-1)}(\operatorname{ch} t-1)^{k}\left\|D_{\lambda}^{k} f\right\|_{p, \lambda} .
$$

Thus Lemma 3.10 is proved.

Lemma 3.11. Let $f \in L_{1, \lambda}, g \in W_{p, \lambda}^{r}, 1 \leq p \leq \infty$. If $f$ is the spectrum of order $\nu$, then their convolution is the spectrum of order $\nu$ and belongs to the class $\mathfrak{M}(\nu, p, \lambda)$.

Theorem 3.1. For $f \in W_{p, \lambda}^{r}, 1 \leq p \leq \infty$ the following inequality is valid

$$
E_{\nu}(f)_{p, \lambda} \lesssim \nu^{-2 r}\left\|D_{\lambda}^{r} f\right\|_{p, \lambda} .
$$

Proof. We can write difference (1.11) in a form

$$
-\Delta_{\text {cht }}^{k} f(\operatorname{ch} x)=-\sum_{i=0}^{k}(-1)^{k-i}\left(\begin{array}{l}
k \\
i
\end{array}\right) A_{\text {cht }}^{i} f(\operatorname{ch} x)
$$




$$
=\sum_{i=1}^{k}(-1)^{i-1}\left(\begin{array}{l}
k \\
i
\end{array}\right) A_{\text {cht }}^{i} f(\operatorname{ch} x)-f(\operatorname{ch} x) .
$$

Let $\varphi($ cht $)$ be the spectrum of order $\nu$ satisfied the condition

$$
\int_{0}^{\infty} \varphi(c h t) s h^{2 \lambda} t \mathrm{~d} t=1
$$

i.e., $\varphi \in \mathfrak{M}(1,1, \lambda)$.

We consider the function

$$
\begin{gathered}
\Phi_{\nu}(\operatorname{ch} x)=\int_{0}^{\infty} \varphi(\operatorname{cht} t)\left(-\Delta_{\operatorname{ch} \frac{t}{\nu}}^{k} f(\operatorname{ch} x)+f(\operatorname{ch} x)\right) \operatorname{sh}^{2 \lambda} t \mathrm{~d} t \\
=\sum_{i=1}^{k}(-1)^{i-1}\left(\begin{array}{l}
k \\
i
\end{array}\right) \int_{0}^{\infty} \varphi(\operatorname{ch} t) A_{c h \frac{t}{\nu}}^{i} f(\operatorname{ch} x) s h^{2 \lambda} t \mathrm{~d} t .
\end{gathered}
$$

Since the operator $A_{c h t}$ is self-adjoint, we have

$$
\begin{gathered}
\int_{0}^{\infty} \varphi(\operatorname{cht}) A_{c h \frac{t}{\nu}}^{i} f(\operatorname{ch} x) s h^{2 \lambda} t \mathrm{~d} t=\nu \int_{0}^{\infty} \varphi(\operatorname{ch} \nu t) A_{\text {cht }}^{i} f(\operatorname{ch} x) s h^{2 \lambda} \nu t \mathrm{~d} t \\
=\int_{0}^{\infty} A_{\text {cht }}\left(A_{\text {cht }}^{i-1} f(\operatorname{ch} x)\right)\left(\frac{\nu \varphi(\operatorname{ch} \nu t) s h^{2 \lambda} \nu t}{s h^{2 \lambda} t}\right) s h^{2 \lambda} t \mathrm{~d} t .
\end{gathered}
$$

Taking into account commutativity of the operator $A_{c h t}$

$$
A_{\text {cht }} A_{\operatorname{ch} x}=A_{\operatorname{chx}} A_{\text {cht }},
$$

we obtain

$$
\begin{aligned}
& \int_{0}^{\infty} A_{\operatorname{cht}}\left(A_{\operatorname{ch} x}^{i-1} \varphi_{\frac{1}{\nu}}(\operatorname{ch} t)\right) f(\operatorname{ch} t) \operatorname{sh}^{2 \lambda} t \mathrm{~d} t \\
= & \int_{0}^{\infty} A_{\operatorname{ch} x}\left(A_{\operatorname{cht} t}^{i-1} \varphi_{\frac{1}{\nu}}\right)(\operatorname{cht} t) f(\operatorname{ch} t) \operatorname{sh}^{2 \lambda} t \mathrm{~d} t .
\end{aligned}
$$

Then from (3.8) we have

$$
\Phi_{\nu}(\operatorname{ch} x)=\left(K_{\nu} * f\right)(\operatorname{ch} x)=\int_{0}^{\infty}\left(A_{\operatorname{ch} x} K_{\nu}\right)(\operatorname{cht} t) f(\operatorname{cht} t) \operatorname{sh}^{2 \lambda} t \mathrm{~d} t,
$$

where

$$
K_{\nu}(\text { cht } t)=\sum_{i=1}^{k}(-1)^{i-1}\left(\begin{array}{c}
k \\
i
\end{array}\right)\left(A_{c h t}^{i-1} \varphi_{\frac{1}{\nu}}(\text { cht } t)\right) .
$$

Taking into account that $\sum_{i=1}^{k}(-1)^{i-1}\left(\begin{array}{c}k \\ i\end{array}\right)=1$, inequality (1.10) and (3.10) we obtain

$$
\left\|K_{\nu}\right\|_{1, \lambda} \leq\left\|\varphi_{\frac{1}{\nu}}\right\|_{1, \lambda}=\|\varphi\|_{1, \lambda}=1
$$

while the dilation operator $\varphi_{\frac{1}{\nu}}$ is an automorphism of $L_{1, \lambda}$.

Since $K_{\nu} \in L_{1, \lambda}$, by Lemma 3.11 the operator $\Phi_{\nu}$ is of the spectrum of order $\nu$, i.e., $\Phi_{\nu} \in \mathfrak{M}(p, \nu, \lambda)$, moreover is an entire function of exponential type (see proof of the Lemma 3.11).

Let $f \in W_{p, \lambda}^{r}$. Using Lemma 3.10 and taking into account (3.7) and (3.8), we obtain

$$
\left\|\Phi_{\nu} f-f\right\|_{p, \lambda} \leq c(\lambda, r) \int_{0}^{\infty}\left\|\Delta_{c h \frac{t}{\nu}}^{r} f(\operatorname{ch}(\cdot))\right\|_{p, \lambda} \varphi(\operatorname{cht} t) s h^{2 \lambda} t \mathrm{~d} t
$$




$$
\begin{aligned}
& \leq c(\lambda, r)\left\|D_{\lambda}^{r} f\right\|_{p, \lambda} \int_{0}^{\infty}\left(\operatorname{ch} \frac{t}{\nu}-1\right)^{r} \varphi(c h t) s h^{2 \lambda} t \mathrm{~d} t \\
& \leq c(\lambda, r)\left\|D_{\lambda}^{r} f\right\|_{p, \lambda} \int_{0}^{\infty}\left(\operatorname{sh} \frac{t}{2 \nu}\right)^{2 r} \varphi(c h t) s h^{2 \lambda} t \mathrm{~d} t \\
& \leq c(\lambda, r) \nu^{-2 r}\left\|D_{\lambda}^{r} f\right\|_{p, \lambda} \int_{0}^{\infty}(s h t)^{2 r+2 \lambda} \varphi(c h t) \mathrm{d} t .
\end{aligned}
$$

In the capacity of the function $\varphi(c h t)$ we take the function

$$
\varphi(\operatorname{ch} t)=\frac{2}{B\left(\lambda+\frac{1}{2}, r+\frac{1}{2}\right)}(\operatorname{ch} t)^{-2 \lambda-2 r-1} .
$$

Then (see [11], p. 361) using of formula

$$
\int_{0}^{\infty} \frac{(\operatorname{sh} x)^{2 \mu-1} \operatorname{ch} x \mathrm{~d} x}{\left(1+a \operatorname{sh}^{2} x\right)^{\nu}}=\frac{1}{2} a^{-\mu} B(\mu, \nu-\mu),(\nu>\mu>0), a>0
$$

at $\mu=\lambda+\frac{1}{2}, \nu=r+\lambda+1$ and $a=1$, we obtain

$$
\int_{0}^{\infty} \varphi(\operatorname{ch} t) \operatorname{sh}^{2 \lambda} t \mathrm{~d} t=\frac{2}{B\left(\lambda+\frac{1}{2}, r+\frac{1}{2}\right)} \int_{0}^{\infty} \frac{\operatorname{sh}^{2 \lambda} t \operatorname{cht} \mathrm{d} t}{(\operatorname{cht})^{2 r+2 \lambda+2}}=1
$$

On the other hand, taking in the formula $(3.12) \mu=r+\lambda+\frac{1}{2}, \nu=r+\lambda+1$ and $a=1$ we obtain

$$
\int_{0}^{\infty} \varphi(\operatorname{cht} t)(\operatorname{sh} t)^{2 \lambda+2 r} \mathrm{~d} t=\int_{0}^{\infty} \frac{(\operatorname{sh} t)^{2 \lambda+2 r} \operatorname{cht} \mathrm{d} t}{(\operatorname{cht} t)^{2 r+2 \lambda+2}}=\frac{1}{2} B\left(r+\lambda+\frac{1}{2}, \frac{1}{2}\right) .
$$

Taking into account (3.13) in (3.11), we have

$$
\left\|\Phi_{\nu} f-f\right\|_{p, \lambda} \leq c(\lambda, r) \nu^{-2 r}\left\|D_{\lambda}^{r} f\right\|_{p, \lambda},
$$

from this the assertion of Theorem 3.12 follows.

Let $K\left(f, t ; L_{p, \lambda} ; W_{p, \lambda}^{m}\right)$ be $K$ - Peetre functional constructing on the spaces $L_{p, \lambda}$ and $W_{p, \lambda}^{m}, f \in$ $L_{p, \lambda}, t>0$. For brevity we take

$$
K_{m}(f, t)_{p, \lambda}:=K\left(f, t^{2 m} ; L_{p, \lambda} ; W_{p, \lambda}^{m}\right)
$$

The modulus $\omega_{k}(f, t)_{p, \lambda}$ of smoothness of order $k$ is defined on Section 1 by the formula (1.12)

$$
\omega_{k}(f, t)_{p, \lambda}=\sup _{0<h \leq t}\left\|\Delta_{c h h}^{k} f\right\|_{p, \lambda}
$$

Proposition 3.1. The modulus of smoothness $\omega_{k}(f, t)_{p, \lambda}, k \in \mathbb{N}, 1 \leq p \leq \infty$ has the following properties:

1. $\omega_{k}(f, t)_{p, \lambda}$ is a non-decreasing function of $t \in[0, \infty)$,

2. $\omega_{k}(f+g, t)_{p, \lambda} \leq \omega_{k}(f, t)_{p, \lambda}+\omega_{k}(g, t)_{p, \lambda}$,

3. $\omega_{k}(f, t)_{p, \lambda} \leq 2^{k}\|f\|_{p, \lambda}$,

4. if $f \in L_{p, \lambda}, 1 \leq p \leq \infty$, then $\lim _{t \rightarrow 0} \omega_{k}(f, t)_{p, \lambda}=0$,

5. if $\nu \leq k$, then $\omega_{k}(f, t)_{p, \lambda} \leq 2^{k-\nu} \omega_{\nu}(f, t)_{p, \lambda}$,

6. if $f \in W_{p, \lambda}^{m}$, then for any $k>m$ the following inequality is valid:

$$
\omega_{k}(f, t)_{p, \lambda} \lesssim t^{2 m} \omega_{k-m}\left(D_{\lambda}^{m} f, t\right)_{p, \lambda}
$$


7. if $f \in W_{p, \lambda}^{k}$, then the inequality is valid:

$$
\omega_{k}(f, t)_{p, \lambda} \leq 2^{k}\left(s h \frac{t}{2}\right)^{2 k}\left\|D_{\lambda}^{k} f\right\|_{p, \lambda} .
$$

We define a family of the operators $P_{\nu}^{n}$ with $\nu>0$ by the formula $P_{v}^{\prime} f=A_{c h \frac{1}{v}}^{\prime} f$,

$$
P_{\nu}^{n} f \equiv P_{\nu} f=A_{c h \frac{1}{\nu}}^{n} f-\sum_{k=1}^{n-1} C_{k}\left(\operatorname{ch} \frac{1}{\nu}\right)\left(A_{\operatorname{ch} \frac{1}{\nu}}^{n-1} D_{\lambda}^{k} f\right), n=2,3, \cdots
$$

Proposition 3.2. Let $f \in \mathfrak{M}(\nu, p, \lambda), 1 \leq p \leq \infty$, $>>0$. Then $P_{\nu} f \in \mathfrak{M}(\nu, p, \lambda)$.

Proposition 3.3. Let $f \in L_{p, \lambda}, 1 \leq p \leq \infty, \nu>0$. For every $t \in\left(0, \frac{1}{\nu}\right)$ the following inequalities hold:

$$
\left\|D_{\lambda}^{m} P_{\nu}(f)\right\|_{p, \lambda} \lesssim\left(\operatorname{sh} \frac{t}{2}\right)^{-2 m}\left\|\Delta_{c h t}^{m} f\right\|_{p, \lambda}
$$

and if $f \in W_{p, \lambda}^{m}$, then

$$
\left\|\Delta_{c h t}^{m} P_{\nu}(f)\right\|_{p, \lambda} \lesssim\left(s h \frac{t}{2}\right)^{2 m}\left\|D_{\lambda}^{m} f\right\|_{p, \lambda} .
$$

Proof. Using the equality (3.2) and also Lemmas 3.2 and 3.3, we obtain

$$
\begin{gathered}
\left(\widehat{\Delta_{c h t}^{m} f}\right)_{P}(\alpha)=C_{m}(\operatorname{ch} t)\left(A_{c h t}^{m\left(D_{\lambda}^{m} f\right)}\right)_{P}(\alpha) \\
=C_{m}(\operatorname{cht} t)\left(\widehat{D_{\lambda}^{m} f}\right)_{P}(\alpha)\left(Q_{\alpha}^{\lambda}(\operatorname{cht} t)\right)^{m} \\
=(\alpha(\alpha+2 \lambda))^{m} C_{m}(\operatorname{ch} t) \hat{f}_{P}(\alpha)\left(Q_{\alpha}^{\lambda}(\operatorname{ch} t)\right)^{m} .
\end{gathered}
$$

Consider the Gegenbauer transform of $D_{\lambda}^{m}\left(P_{\nu} f\right)$. Using (2.6) and (3.18) we write

$$
\begin{aligned}
& \left(\widehat{D_{\lambda}^{m} P_{\nu}} f\right)_{P}(\alpha)=(\alpha(\alpha+2 \lambda))^{m}\left(\widehat{P_{\nu} f}\right)_{P}(\alpha) \\
& =\frac{(\alpha(\alpha+2 \lambda))^{m} C_{m}(\operatorname{ch} t){\widehat{P_{\nu} f}}_{P}(\alpha)\left(Q_{\alpha}^{\lambda}(\operatorname{ch} t)\right)^{m}}{C_{m}(\operatorname{ch} t)\left(Q_{\alpha}^{\lambda}(\operatorname{cht} t)^{m}\right.}=\frac{\left(\Delta_{\operatorname{cht} P_{\nu}}^{m}\right)_{P}(\alpha)}{C_{m}(\operatorname{cht} t)\left(Q_{\alpha}^{\lambda}(\operatorname{ch} t)\right)^{m}} .
\end{aligned}
$$

Using the equality (3.19) inverse Gegenbauer transform and taking into account we have

$$
D_{\lambda}^{m} P_{\nu} f(\operatorname{ch} x)=\frac{\Delta_{c h t}^{m} P_{\nu} f(\operatorname{ch} x)}{C_{m}(\operatorname{ch} t)\left(Q_{\alpha}^{\lambda}(\operatorname{ch} t)\right)^{m}}
$$

From (1.3) it follows that the function $Q_{\alpha}^{\lambda}(\operatorname{ch} t)$ is increasing. Moreover

$$
\lim _{t \rightarrow \infty} Q_{\alpha}^{\lambda}(\operatorname{ch} t)=\infty
$$

and from the formula (see [9], p. 1934)

$$
Q_{\alpha}^{\lambda}(\operatorname{cht})=F\left(-\alpha, \alpha+2 \lambda ; \lambda+\frac{1}{2}, \operatorname{sh}^{2} \frac{t}{2}\right), \quad 0 \leq \operatorname{sh} \frac{t}{2} \leq 1
$$

This function takes its minimal value at $t=0$ (see [17], p. 1053). We have the following

$$
\min Q_{\alpha}^{\lambda}(\operatorname{ch} t)=Q_{\alpha}^{\lambda}(1)=F\left(-\alpha, \alpha+2 \lambda ; \lambda+\frac{1}{2} ; 0\right)=1 .
$$


On the other hand (see [14], Lemma 5)

$$
c_{1}(m, \lambda)\left(\operatorname{sh} \frac{t}{2}\right)^{2 m} \leq C_{m}(\operatorname{ch} t) \leq c_{2}(m, \lambda)\left(\operatorname{sh} \frac{t}{2}\right)^{2 m} .
$$

Taking into account (3.21) and (3.22) in (3.20), we have

$$
\left\|D_{\lambda}^{m}\left(P_{\nu} f\right)\right\|_{p, \lambda} \lesssim\left(s h \frac{t}{2}\right)^{-2 m}\left\|\Delta_{c h t}^{m} P_{\nu} f\right\|_{p, \lambda} .
$$

From Lemmas 3.7 and 3.1 it follows that

$$
P_{\nu} f(\operatorname{ch} x)=f(\operatorname{ch} x)+\triangle_{\operatorname{ch} \frac{1}{\nu}}^{n} f(\operatorname{ch} x)
$$

then from (1.10) we have

$$
\left\|P_{\nu} f\right\|_{p, \lambda}=\left\|f+\triangle_{c h \frac{1}{\nu}}^{n} f\right\|_{p, \lambda} \leq\left(2^{n}+1\right)\|f\|_{p, \lambda} .
$$

From this taking into account the commutativity of the operator $A_{c h t}$, we obtain

$$
\left\|D_{\lambda}^{m}\left(P_{\nu} f\right)\right\|_{p, \lambda} \lesssim\left(\operatorname{sh} \frac{t}{2}\right)^{-2 m}\left\|P_{\nu}\left(\Delta_{c h t}^{m} f\right)\right\|_{p, \lambda} \lesssim\left(\operatorname{sh} \frac{t}{2}\right)^{-2 m}\left\|\Delta_{c h t}^{m} f\right\|_{p, \lambda}
$$

then the inequality (3.16) is proved.

Now, let $f \in W_{p, \lambda}^{m}$. From (3.18) we have

$$
\left.\left(\Delta_{c h t}^{m} \widehat{\left(P_{\nu}\right.} f\right)\right)_{P}(\alpha)=C_{m}(\operatorname{cht})\left(A_{c h t}^{m} \widehat{D_{\lambda}^{m}\left(P_{\nu} f\right)}\right)_{P}(\alpha) .
$$

Applying the inverse transformation $F_{P}^{-1}$ to equality (3.24), we obtain

$$
\begin{aligned}
& \Delta_{c h t}^{m} P_{\nu} f=C_{m}(\operatorname{ch} t) A_{c h t}^{m} D_{\lambda}^{m}\left(P_{\nu} f\right) \\
= & C_{m}(\operatorname{ch} t) A_{c h t}^{m} D_{\lambda}^{m}\left(f+\Delta_{c h \frac{1}{\nu}}^{m} f\right) \\
= & C_{m}(\operatorname{ch} t) A_{c h t}^{m}\left(D_{\lambda}^{m} f+\Delta_{c h \frac{1}{\nu}}^{m} D_{\lambda}^{m} f\right),
\end{aligned}
$$

from where taking into account (3.22) and (1.10), we have

$$
\left\|\Delta_{c h t}^{m} P_{\nu} f\right\|_{p, \lambda} \lesssim\left(s h \frac{t}{2}\right)^{2 m}\left\|A_{c h t}^{m} D_{\lambda}^{m} f\right\|_{p, \lambda} \lesssim\left(s h \frac{t}{2}\right)^{2 m}\left\|D_{\lambda}^{m} f\right\|_{p, \lambda},
$$

then the inequality (3.17) is proved.

Thus Proposition 3.3 is proved.

Here is a useful corollary that follows from inequality (3.16) and the definition of the modulus of smoothness.

Corollary 3.1. For $f \in L_{p, \lambda}, 1 \leq p \leq \infty, \nu>0, m \in \mathbb{N}$ the following inequality holds

$$
\left\|D_{\lambda}^{m}\left(P_{\nu} f\right)\right\|_{p, \lambda} \lesssim \nu^{2 m} \omega_{m}\left(f, \frac{1}{\nu}\right)_{p, \lambda} .
$$

Then it follows from the fact that

$$
\frac{1}{s h^{2 m} \frac{1}{2 \nu}} \leq \frac{1}{\left(\frac{1}{2 \nu}\right)^{2 m}}=4^{m} \nu^{2 m} .
$$

The following proposition and corollary can be regarded as analogous of the classical Boas inequality (see [41], p. 266). 
Proposition 3.4. Let $f \in W_{p, \lambda}^{m}, 1 \leq p \leq \infty, \nu>0$. For every numbers $\delta$ and $t$ such that $0<\delta<t<\frac{1}{\nu}$ the following inequality

$$
\left(\operatorname{sh} \frac{\delta}{2}\right)^{-2 m}\left\|\Delta_{c h \delta}^{m} f\right\|_{p, \lambda} \lesssim\left(\operatorname{sh} \frac{t}{2}\right)^{-2 m}\left\|\Delta_{c h t}^{m} f\right\|_{p, \lambda}
$$

is valid.

Proof. From (3.2) we have:

$$
\Delta_{c h \delta}^{m} f(\operatorname{ch} x)=C_{m}(\operatorname{ch} \delta) A_{c h \delta}^{m} D_{\lambda}^{m} f(\operatorname{ch} x)
$$

and

$$
\Delta_{c h t}^{m} f(\operatorname{ch} x)=C_{m}(\operatorname{cht}) A_{c h t}^{m} D_{\lambda}^{m} f(\operatorname{ch} x) .
$$

Then the following equalities follow:

$$
\begin{aligned}
\left\|\Delta_{c h \delta}^{m} f\right\|_{p, \lambda} & =C_{m}(\operatorname{ch} \delta)\left\|A_{c h \delta}^{m} D_{\lambda}^{m} f\right\|_{p, \lambda}, \\
\left\|\Delta_{c h t}^{m} f\right\|_{p, \lambda} & =C_{m}(\operatorname{cht} t)\left\|A_{c h t}^{m} D_{\lambda}^{m} f\right\|_{p, \lambda} .
\end{aligned}
$$

From the continuity of the operator $A_{c h t}$ in $W_{p, \lambda}^{m}$, we have

$$
\lim _{t \rightarrow 0}\left\|\Delta_{c h t}^{m} f / C_{m}(\operatorname{cht} t)\right\|_{p, \lambda}=\lim _{\delta \rightarrow 0}\left\|\Delta_{c h \delta}^{m} f / C_{m}(\operatorname{ch} \delta)\right\|_{p, \lambda}=\left\|D_{\lambda}^{m} f\right\|_{p, \lambda} .
$$

Since $t \rightarrow 0$ involve $\delta \rightarrow 0$ that

$$
\left\|\Delta_{c h t}^{m} f\right\|_{p, \lambda} / C_{m}(\text { cht } t) \sim\left\|\Delta_{c h \delta}^{m} f\right\|_{p, \lambda} / C_{m}(\operatorname{ch} \delta) \text { ïð̀ } t \rightarrow 0, \delta \rightarrow 0
$$

from this it follows that $0<\delta<t<\frac{1}{\nu}$

$$
C_{m}(\operatorname{ch} t)\left\|\Delta_{c h \delta}^{m} f\right\|_{p, \lambda} \sim C_{m}(\operatorname{ch} \delta)\left\|\Delta_{c h t}^{m} f\right\|_{p, \lambda} .
$$

Taking into account (3.22), we obtain (3.27).

Thus Proposition 3.4 is proved.

Corollary 3.2. Let $f \in W_{p, \lambda}^{m}, 1 \leq p \leq \infty, \nu>0, m \in \mathbb{N}$. For any $t \in\left(0, \frac{1}{\nu}\right]$ inequality

$$
\left\|D_{\lambda}^{m} f\right\|_{p, \lambda} \lesssim\left(s h \frac{t}{2}\right)^{-2 m}\left\|\Delta_{c h t}^{m} f\right\|_{p, \lambda}
$$

is valid. Taking into account of the continuity of operator $A_{c h t}$ in $W_{p, \lambda}^{m}$ and also relation (see [14], Lemma 5)

$$
C_{m}(\operatorname{ch} t) \sim c(m, \lambda)(\operatorname{ch} t-1)^{m} \text { at } t \rightarrow 0,
$$

where

$$
c(m, \lambda)=\frac{1}{m !(2 \lambda+1)(2 \lambda+3) \ldots .(2 \lambda+2 m-1)} .
$$

From (3.28) we have

$$
\lim _{\delta \rightarrow 0}\left\|\frac{\Delta_{c h \delta}^{m} f}{C_{m}(\operatorname{ch\delta } \delta)}\right\|_{p, \lambda}=\lim _{\delta \rightarrow 0}\left\|\Delta_{c h \delta}^{m}\left(D_{\lambda}^{m} f\right)\right\|_{p, \lambda}=\left\|D_{\lambda}^{m} f\right\|_{p, \lambda} .
$$

If we take limit as $\delta \rightarrow 0$ in (3.27), then we obtain (3.30).

Inequality (3.30) is an analogue of the classical Nikol'skii-Stechkin inequality (see [29], [41]).

Taking into account that $s h \frac{t}{2} \geq \frac{t}{2}$ from (3.30) we obtain

$$
\left\|D_{\lambda}^{m} f\right\|_{p, \lambda} \lesssim\left(\frac{2}{t}\right)^{2 m} 2^{m}\|f\|_{p, \lambda} .
$$


Putting here $t=\frac{1}{\nu}$, we have the inequality

$$
\left\|D_{\lambda}^{m} f\right\|_{p, \lambda} \lesssim \nu^{2 m}\|f\|_{p, \lambda} .
$$

From this at $m=1$ the assertion of Theorem 1.3 follows.

Proposition 3.5. Let $f \in W_{p, \lambda}^{m}, 1 \leq p \leq \infty$, and $\nu>0$. For any number $\delta$ and $t$ such that $0<\delta<t<\frac{1}{\nu}$ the following inequality holds:

$$
\left(\operatorname{sh} \frac{\delta}{2}\right)^{2 m}\left\|\Delta_{c h \delta}^{m} P_{\nu} f\right\|_{p, \lambda} \lesssim\left(s h \frac{t}{2}\right)^{-2 m}\left\|\Delta_{c h \delta}^{m} P_{\nu} f\right\|_{p, \lambda} .
$$

Proof. Changing into (3.28) $f$ by $P_{\nu}(f)$ we have

$$
\Delta_{c h \delta}^{m} P_{\nu} f(\operatorname{ch} x)=C_{m}(\operatorname{ch} \delta) A_{c h \delta}^{m} D_{\lambda}^{m} P_{\nu} f(\operatorname{ch} x) .
$$

From here taking into account the inequalities (3.23) and (3.30), we obtain

$$
\begin{aligned}
& \left\|\Delta_{c h \delta}^{m} P_{\nu} f\right\|_{p, \lambda} \leq C_{m}(\operatorname{ch} \delta)\left\|D_{\lambda}^{m} P_{\nu} f\right\|_{p, \lambda} \\
& \lesssim\left(\operatorname{sh} \frac{\delta}{2}\right)^{2 m}\left(\operatorname{sh} \frac{t}{2}\right)^{-2 m}\left\|\Delta_{c h t}^{m} P_{\nu} f\right\|_{p, \lambda},
\end{aligned}
$$

from here (3.32) follows.

Thus Proposition 3.5 is proved.

Lemma 3.12. Let $g \in W_{p, \lambda}^{m}, 1 \leq p \leq \infty, t>0$. Then the following estimate holds:

$$
\omega_{m}(g, t)_{p, \lambda} \lesssim\left(s h \frac{t}{2}\right)^{2 m}\left\|D_{\lambda}^{m} g\right\|_{p, \lambda} .
$$

Proof. Let $u \in(0, t], \nu=\frac{1}{t}$. From equality

$$
\Delta_{c h u}^{m} g=\Delta_{c h u}^{m}\left(g-P_{\nu} f\right)+\Delta_{c h u}^{m} P_{\nu} f,
$$

using consecutive the inequalities (1.10), (3.17) and Theorem 3.1 we obtain

$$
\begin{gathered}
\left\|\Delta_{c h u}^{m} g\right\|_{p, \lambda} \leq 2^{m}\left\|g-P_{\nu} f\right\|_{p, \lambda}+\left\|\Delta_{c h u}^{m} P_{\nu} f\right\|_{p, \lambda} \\
\leq 2^{m} E_{\nu}(f)_{p, \lambda}+\left(s h \frac{u}{2}\right)^{2 m}\left\|D_{\lambda}^{m} g\right\|_{p, \lambda} \lesssim t^{2 m}\left\|D_{\lambda}^{m} g\right\|_{p, \lambda} \\
+\left(s h \frac{t}{2}\right)^{2 m}\left\|D_{\lambda}^{m} g\right\|_{p, \lambda} \lesssim\left(s h \frac{t}{2}\right)^{2 m}\left\|D_{\lambda}^{m} g\right\|_{p, \lambda},
\end{gathered}
$$

from this the inequality (3.33) follows .

Thus Lemma 3.12 is proved.

Proposition 3.6. For any function $f \in L_{p, \lambda}, 1 \leq p \leq \infty$ and number $m \in \mathbb{N}, t>0$ the inequality is valid:

$$
\omega_{m}(f, t)_{p, \lambda} \lesssim K_{m}(f, t) .
$$

Proof. Let $g \in W_{p, \lambda}^{m}$. Using smoothness property of the modulus and Lemma 3.12, we obtain

$$
\begin{gathered}
\omega_{m}(f, t)_{p, \lambda} \leq \omega_{m}(f-g, t)_{p, \lambda}+\omega_{m}(g, t)_{p, \lambda} \\
\lesssim\|f-g\|_{p, \lambda}+\left(s h \frac{t}{2}\right)^{2 m}\left\|D_{\lambda}^{m} g\right\|_{p, \lambda} \lesssim\|f-g\|_{p, \lambda}+t^{2 m}\left\|D_{\lambda}^{m} g\right\|_{p, \lambda},
\end{gathered}
$$

as $s h t \sim t$ at $t \rightarrow 0$. Taking the infimum over all $g \in W_{p, \lambda}^{m}$, we obtain inequality (3.34).

Thus Proposition 3.6 is proved. 


\section{Proof of the Theorems 1.1 and 1.2.}

We now prove an analogue of Jackson's direct theorem, from which we shall deduce Theorem 1.1.

Theorem 4.1. For $f \in L_{p, \lambda}, 1 \leq p \leq \infty, k \in \mathbb{N}, \nu>0$ the following inequality holds

$$
E_{\nu}(f)_{p, \lambda} \leq \omega_{k}\left(f, \frac{1}{\nu}\right)_{p, \lambda}
$$

Proof. Since

$$
E_{\nu}(f)_{p, \lambda} \leq\left\|f-P_{\nu} f\right\|_{p, \lambda}, \quad\left\|\Delta_{c h \frac{1}{\nu}}^{k} f\right\|_{p, \lambda} \leq \omega_{k}\left(f, \frac{1}{\nu}\right)_{p, \lambda},
$$

then it is enough to prove the inequality

$$
\left\|f-P_{\nu} f\right\|_{p, \lambda} \leq\left\|\Delta_{c h \frac{1}{\nu}}^{k} f\right\|_{p, \lambda}
$$

for prove (4.1). From (3.23) we have

$$
P_{\nu} f(\operatorname{ch} x)=\Delta_{c h \frac{1}{\nu}}^{n} f(\operatorname{ch} x)+f(\operatorname{ch} x)
$$

from this (4.2) follows. Thus Theorem 4.1 is proved.

Proof of Theorem 1.1. Let $f \in W_{p, \lambda}^{m}$. Taking in Theorem $4.1 k+m$ instead of $k$, we obtain the following inequality

$$
E_{\nu}(f)_{p, \lambda} \leq \omega_{k+m}\left(f, \frac{1}{\nu}\right)_{p, \lambda} .
$$

From the smoothness property of the modulus it follows that

$$
\omega_{k+m}(f, t)_{p, \lambda} \lesssim \nu^{-2 m} \omega_{k}\left(D_{\lambda}^{m} f, \frac{1}{\nu}\right)_{p, \lambda},
$$

from where we have

$$
E_{\nu}(f)_{p, \lambda} \lesssim \nu^{-2 m} \omega_{k}\left(D_{\lambda}^{m} f, \frac{1}{\nu}\right)_{p, \lambda}
$$

Thus the proof of the theorem is completed.

Proof of Theorem 1.2. We ought to justice of the correlation

$$
K_{m}(f, t) \simeq \omega_{m}(f, t)_{p, \lambda}, \quad t \rightarrow 0 .
$$

If we take $g=P_{\nu} f, \nu>0$, then from the definition of $K_{m}(f, t)$ it follows that

$$
K_{m}(f, t) \leq\left\|f-P_{\nu} f\right\|_{p, \lambda}+t^{2 m}\left\|D_{\lambda}^{m}\left(P_{\nu} f\right)\right\|_{p, \lambda} .
$$

Using inequality (3.26) in (4.5), we obtain

$$
K_{m}(f, t) \lesssim E_{\nu}(f)_{p, \lambda}+\nu^{2 m} \omega_{m}\left(f, \frac{1}{\nu}\right)_{p, \lambda} \lesssim\left(1+(t \nu)^{2 m}\right) \omega_{m}\left(f, \frac{1}{\nu}\right)_{p, \lambda} .
$$

Putting in (4.6) $\nu=\frac{1}{t}$, we obtain

$$
K_{m}(f, t) \lesssim \omega_{m}(f, t)_{p, \lambda} .
$$

The correlation (4.4) follows from (3.34) and (4.7).

Theorem 1.2 is proved.

In the following proposition which will be derived from Theorem 1.2 , we state for $\omega_{m}(f, t)_{p, \lambda}$. 
Proposition 4.1. For $f \in L_{p, \lambda}, t>0, \delta>0$ the relation

$$
\omega_{m}(f, \delta t)_{p, \lambda} \lesssim \max \left\{1, \delta^{2 m}\right\} \omega_{m}(f, t)_{p, \lambda}
$$

is valid.

Proof. Let $0<\delta \leq 1$, then the definition of functional we have

$$
\begin{gathered}
K_{m}(f, \delta t)=\inf \left\{\|f-g\|_{p, \lambda}+(\delta t)^{m}\left\|D_{\lambda}^{m} f\right\|_{p, \lambda}: g \in W_{p, \lambda}^{m}\right\} \\
\leq \inf \left\{\|f-g\|_{p, \lambda}+t^{m}\left\|D_{\lambda}^{m} f\right\|_{p, \lambda}: g \in W_{p, \lambda}^{m}\right\} .
\end{gathered}
$$

And at $\delta>1$

$$
\begin{aligned}
& K_{m}(f, \delta t) \leq \inf \left\{\delta^{2 m}\|f-g\|_{p, \lambda}+\delta^{2 m} t^{m}\left\|D_{\lambda}^{m} f\right\|_{p, \lambda}: g \in W_{p, \lambda}^{m}\right\} \\
= & \delta^{2 m} \inf \left\{\|f-g\|_{p, \lambda}+t^{m}\left\|D_{\lambda}^{m} f\right\|_{p, \lambda}: g \in W_{p, \lambda}^{m}\right\}=\delta^{2 m} K_{m}(f, t) .
\end{aligned}
$$

From this and (4.4), (4.8) follows.

\section{Nikol'skii-Besov Space and Their Approximation Characteristics. The Embeddings Theorems}

In this section we deal with the spaces $H_{p, \lambda}^{r}$ and $B_{p, q, \lambda}^{r}$ of Nikol'skii-Besov type and describe them in terms of the best approximation by functions on class $\mathfrak{M}(\nu, p, \lambda)$. The definition of spaces $H_{p, \lambda}^{r}$ and $B_{p, q, \lambda}^{r}$ can be found in Section 1. We will show that these spaces are Banach spaces. For these at first we will prove the completeness of the space $L_{p, \lambda}$.

Proposition 5.1. The space $L_{p, \lambda}, 1 \leq p<\infty$ is complete.

Proof. Let $\left\{f_{n}(\operatorname{ch} x)\right\}, n \in N$ be the sequence of functions in the space $L_{p, \lambda}[0, \infty]$ such that

$$
\left\|f_{n}-f_{m}\right\|_{p, \lambda} \rightarrow 0 \quad \text { as } n, m \rightarrow \infty
$$

From this it follows that

$$
\int_{0}^{\infty}\left|f_{n}(\operatorname{ch} x)-f_{m}(\operatorname{ch} x)\right|^{p} \operatorname{sh}^{2 \lambda} x \mathrm{~d} x \rightarrow 0 \quad \text { as } n, m \rightarrow \infty .
$$

From the sequence $\left\{f_{n}(\operatorname{ch} x)\right\}$ we choose a subsequence $\left\{f_{n_{k}}(\operatorname{ch} x)\right\}$ such that

$$
A=\sum_{k=1}^{\infty} \int_{0}^{\infty}\left|f_{n_{k+1}}(\operatorname{ch} x)-f_{n_{k}}(\operatorname{ch} x)\right|^{p} \operatorname{sh}^{2 \lambda} x \mathrm{~d} x<\infty .
$$

Then, for any $\ell \in N$, we can write

$$
\begin{aligned}
& \int_{0}^{\infty}\left(\sum_{k=1}^{\ell}\left|f_{n_{k+1}}(\operatorname{ch} x)-f_{n_{k}}(\operatorname{ch} x)\right|\right)^{p} \operatorname{sh}^{2 \lambda} x \mathrm{~d} x \\
\leq & \sum_{k=1}^{\ell} \int_{0}^{\infty}\left|f_{n_{k+1}}(\operatorname{ch} x)-f_{n_{k}}(\operatorname{ch} x)\right|^{p} \operatorname{sh}^{2 \lambda} \mathrm{d} x \leq A .
\end{aligned}
$$

By the Lebesgue's dominated convergence theorem as $\ell \rightarrow \infty$, we obtain

$$
\int_{0}^{\infty}\left(\sum_{k=1}^{\infty}\left|f_{n_{k+1}}(\operatorname{ch} x)-f_{n_{k}}(\operatorname{ch} x)\right|\right)^{p} \operatorname{sh}^{2 \lambda} x \mathrm{~d} x \leq A .
$$


Therefore,

$$
\sum_{k=1}^{\infty}\left|f_{n_{k+1}}(\operatorname{ch} x)-f_{n_{k}}(\operatorname{ch} x)\right|<\infty \quad \text { for a.e. } x \in[0, \infty) .
$$

Hence, in view of the completeness of real axis, the sequence $\left\{f_{n_{k}}(\operatorname{ch} x)\right\}$ is convergent for a.e. $x \in[0, \infty)$.

Denote by $f$ the measurable function defined as

$$
f(\operatorname{ch} x)= \begin{cases}\lim _{k \rightarrow \infty} f_{n_{k}}(\operatorname{ch} x) & \text { for a.e. } x \in[0, \infty), \\ 0, & \text { otherwise }\end{cases}
$$

Thus we have proved that $f_{n_{k}}(\operatorname{ch} x) \rightarrow f(\operatorname{ch} x)$ for a.e. $x \in[0, \infty)$. By Minkowski inequality and inequality (5.1), for all $k \in N$ we have

$$
\begin{aligned}
\left\|f_{n_{k}}\right\|_{p, \lambda} & =\left\|f_{n_{1}}+\sum_{\nu=1}^{k-1}\left(f_{n_{\nu+1}}-f_{n_{\nu}}\right)\right\|_{p, \lambda} \leq\left\|\left|f_{n_{1}}\right|+\sum_{\nu=1}^{k-1}\left|f_{n_{\nu+1}}-f_{n_{\nu}}\right|\right\|_{p, \lambda} \\
& \leq\left\|f_{n_{1}}\right\|_{p, \lambda}+\left\|\sum_{\nu=1}^{k-1}\left|f_{n_{\nu+1}}-f_{n_{\nu}}\right|\right\|_{p, \lambda} \leq\left\|f_{n_{1}}\right\|_{p, \lambda}+A .
\end{aligned}
$$

Passing to the limit as $k \rightarrow \infty$, we obtain that

$$
\left\|f_{n_{k}}\right\|_{p, \lambda} \leq \text { const, } \quad \text { a.e. } f \in L_{p, \lambda}[0, \infty) .
$$

Let us now show that

$$
\left\|f-f_{n_{k}}\right\|_{p, \lambda} \rightarrow 0 \text { as } k \rightarrow \infty .
$$

Indeed taking into account (5.1) we have

$$
\begin{gathered}
\left\|f-f_{n_{k}}\right\|_{p, \lambda}=\left\|\sum_{\nu=k}^{\infty}\left(f_{n_{\nu+1}}-f_{n_{\nu}}\right)\right\|_{p, \lambda} \\
\leq\left\|\sum_{\nu=k}^{\infty}\left|f_{n_{\nu+1}}-f_{n_{\nu}}\right|\right\|_{p, \lambda} \leq\left\|\sum_{\nu=1}^{\infty}\left|f_{n_{\nu+1}}-f_{n_{\nu}}\right|\right\|_{p, \lambda} \leq A^{\frac{1}{p}} .
\end{gathered}
$$

By the Lebesgue's dominated convergence theorem

$$
\left\|f-f_{n_{k}}\right\|_{p, \lambda} \rightarrow 0 \text { as } k \rightarrow \infty .
$$

Finally, we prove that the sequence $\left\{f_{n_{k}}(\operatorname{ch} x)\right\}$ is convergent in $L_{p, \lambda}$. Since the sequence $\left\{f_{n_{k}}(\operatorname{ch} x)\right\}$ is fundamental, it follows that for a given $\varepsilon>0$ there exists a number $N_{\varepsilon}$ such that, for all $m, n>N_{\varepsilon}$

$$
\left\|f_{n}-f_{m}\right\|_{p, \lambda}<\frac{\varepsilon}{2} .
$$

Choose the number $n_{k}>N_{\varepsilon}$ so that

$$
\left\|f-f_{n_{k}}\right\|_{p, \lambda}<\frac{\varepsilon}{2} .
$$

But then we have

$$
\left\|f-f_{n}\right\|_{p, \lambda} \leq\left\|f-f_{n_{k}}\right\|_{p, \lambda}+\left\|f_{n}-f_{n_{k}}\right\|_{p, \lambda}<\varepsilon .
$$

The proof of Proposition 5.1 is complete.

Now we prove the completeness of the space $H_{p, \lambda}^{r}$. By inequality (3.31) for all $f_{n}, f_{m} \in H_{p, \lambda}^{r}$ we have

$$
\left\|D_{\lambda}^{s} f_{n}-D_{\lambda}^{s} f_{m}\right\|_{p, \lambda}=\left\|D_{\lambda}^{s}\left(f_{n}-f_{m}\right)\right\|_{p, \lambda} \lesssim\left\|f_{n}-f_{m}\right\|_{p, \lambda} .
$$

We will show that $H_{p, \lambda}^{r}$ is a Banach space with the norm

$$
\|f\|_{H_{p, \lambda}^{r}}=\|f\|_{p, \lambda}+\sup _{\delta>0} \frac{\omega_{k}\left(D_{\lambda}^{s} f, \delta\right)_{p, \lambda}}{\delta^{r-2 s}} .
$$


We can write

$$
\left\|f_{n}-f_{m}\right\|_{H_{p, \lambda}^{r}}=\left\|f_{n}-f_{m}\right\|_{p, \lambda}+\sup _{\delta>0} \frac{\omega_{k}\left(D_{\lambda}^{s} f_{n}-D_{\lambda}^{s} f_{m}, \delta\right)_{p, \lambda}}{\delta^{r-2 s}} .
$$

Since (see Proposition 3.1 property 3 )

$$
\omega_{k}\left(D_{\lambda}^{s} f, \delta\right)_{p, \lambda} \leq 2^{k}\left\|D_{\lambda}^{s} f\right\|_{p, \lambda},
$$

then

$$
\frac{\omega_{k}\left(D_{\lambda}^{s} f, \delta\right)_{p, \lambda}}{\delta^{r-2 s}} \lesssim\left\|D_{\lambda}^{s} f\right\|_{p, \lambda}
$$

At $\delta \geq 1$ the inequality (5.6) is obvious, but for $0<\delta<1$ it is submitted to condition $2^{m-1}<\frac{1}{\delta}<2^{m}$ (evidently, $m \geq 1$ ). Taking into account (5.2) and (5.6) in (5.4), we obtain

$$
\left\|f_{n}-f_{m}\right\|_{H_{p, \lambda}^{r}} \leq\left\|f_{n}-f_{m}\right\|_{p, \lambda}+\left\|D_{\lambda}^{s} f_{n}-D_{\lambda}^{s} f_{m}\right\|_{p, \lambda} \lesssim\left\|f_{n}-f_{m}\right\|_{p, \lambda} .
$$

Let for every $\varepsilon>0,\left\|f_{n}-f_{m}\right\|_{p, \lambda}<\varepsilon$, at $n, m>N$. Then from (5.7) it follows that

$$
\left\|f_{n}-f_{m}\right\|_{H_{p, \lambda}^{r}}<\varepsilon, \text { for } n, m>N .
$$

Because of completeness of the space $L_{p, \lambda}$ we are able to write

$$
\left\|f_{n}-f\right\|_{H_{p, \lambda}^{r}} \leq\left\|f_{n}-f\right\|_{p, \lambda}<\varepsilon, \text { ï̀̀ } n>N,
$$

from this the completeness of the space $H_{p, \lambda}^{r}$ follows.

Now we show that $B_{p, q, \lambda}^{r}$ is a Banach space. Let for the class $B_{p, q, \lambda}^{r}$ for every $\varepsilon>0$

$$
\begin{gathered}
\left\|f_{n}-f_{m}\right\|_{B_{p, q, \lambda}^{r}}=\left\|f_{n}-f_{m}\right\|_{p, \lambda}+\left(\int_{0}^{\infty} \frac{\omega_{k}\left(D_{\lambda}^{s} f_{n}-D_{\lambda}^{s} f_{m}, \delta\right)_{p, \lambda}^{q}}{\delta^{(r-2 s) q}} \frac{\mathrm{d} \delta}{\delta}\right)^{\frac{1}{q}}<\varepsilon \\
(n, m>N, k \geq 1 ; s=1,2, \ldots) .
\end{gathered}
$$

By the Lebesgue's dominated convergence theorem as $m \rightarrow \infty$, we obtain

$$
\left\|f_{n}-f\right\|_{B_{p, q, \lambda}^{r}}=\left\|f_{n}-f\right\|_{p, \lambda}+\left(\int_{0}^{\infty} \frac{\omega_{k}\left(D_{\lambda}^{s} f_{n}-D_{\lambda}^{s} f, \delta\right)_{p, \lambda}}{\delta^{(r-2 s) q}} \frac{\mathrm{d} \delta}{\delta}\right)^{\frac{1}{q}}<\varepsilon
$$

for $n>N$. Then the completeness of the space $B_{p, q, \lambda}^{r}$ follows. For these spaces the invested theorems are obtained. Let $c_{1}, c_{2}, \ldots$ denote positive constants that are different in different places and can depend on some parameters.

To prove inverse theorems of approximation theory we use inequalities of Bernstein type. Moreover for the inequality (3.31) we need still one inequality of Bernstein type.

Lemma 5.1. For $\Phi \in \mathfrak{M}(\nu, p, \lambda)$ and $t>0$ the following inequality holds

$$
\left\|\Delta_{c h t}^{m} \Phi\right\|_{p, \lambda} \lesssim(\nu t)^{2 m}\|\Phi\|_{p, \lambda} .
$$

Proof. From inequalities (3.3) and (3.31) it follows that

$$
\left\|\Delta_{c h t}^{m} \Phi\right\|_{p, \lambda} \lesssim t^{2 m}\left\|D_{\lambda}^{m} \Phi\right\|_{p, \lambda} \lesssim(t \nu)^{2 m}\|\Phi\|_{p, \lambda} .
$$

Inequality (5.9) implies that (5.8) holds. Thus Lemma 5.1 is proved.

In what follows $k, s \in \mathbb{Z}_{+}, r>0,2 k>r-2 s, \nu \geq 1,1 \leq q \leq \infty$.

We put for brevity $\|\cdot\|:=\|\cdot\|_{p, \lambda}$. 
Proof of Theorem 1.4 the same now of the Theorem 1.2 in [13].

Let

$$
\tilde{h}_{p, \lambda}^{r}(f):=\sup _{\nu \geq 1} \nu^{r} E_{\nu}(f)_{p, \lambda} .
$$

It follows from Theorem 1.4 that $f \in L_{p, \lambda}$ belongs to $H_{p, \lambda}^{r}$ if and only if $\tilde{h}_{p, \lambda}^{r}(f)<\infty$, and the norm in $H_{p, \lambda}^{r}$ is equivalent to the norm

$$
{ }^{1}\|f\|_{H_{p, \lambda}^{r}}:=\|f\|_{p, \lambda}+\tilde{h}_{p, \lambda}^{r}(f) .
$$

In particular for different $k, s$ are such that $2 k>r-2 s>0$, the spaces $H_{p, \lambda}^{r}$ coincide and their norms are equivalent.

In the following theorem we obtain various equivalent norms in the spaces $B_{p, q, \lambda}^{r}$. In particular some of them will follow Theorem 1.5. As before let $r>0, a>1$ be real number, $k$ and $s$ be arbitrary non negative integers such that $2 k>r-2 s>0$. We shall say that a function $f(x)$ belongs to the space ${ }^{j} B_{p, q, \lambda}^{r}, j=1,2,3,4$, if $f \in L_{p, \lambda}$ and the seminorm ${ }^{j} b_{p, q, \lambda}^{r}$, is finite, where

$$
\begin{aligned}
& { }^{1} b_{p, q, \lambda}^{r}(f):=\left\{\begin{array}{lcc}
\left(\int_{0}^{\infty} \frac{\left(\omega_{k}\left(D_{\lambda}^{s} f, \delta\right)_{p, \lambda}\right)^{q}}{\delta^{(r-2 s) q}} \frac{\mathrm{d} \delta}{\delta}\right)^{\frac{1}{q}} & \text { if } & 1 \leq q<\infty, \\
\sup _{\delta>0} \frac{\omega_{k}\left(D_{\lambda}^{s} f, \delta\right)_{p, \lambda}}{\delta^{r-2 s}} & \text { if } & q=\infty,
\end{array}\right. \\
& { }^{2} b_{p, q, \lambda}^{r}(f):=\left\{\begin{array}{lcr}
\left(\int_{0}^{a} \frac{\left(\omega_{k}\left(D_{\lambda}^{s} f, \delta\right)_{p, \lambda}\right)^{q}}{\delta^{(r-2 s) q}} \frac{\mathrm{d} \delta}{\delta}\right)^{\frac{1}{q}} & \text { if } & 1 \leq q<\infty, \\
\sup _{0<\delta \leq a} \delta^{-(r-2 s)} \omega_{k}\left(D_{\lambda}^{s} f, \delta\right)_{p, \lambda} & \text { if } & q=\infty,
\end{array}\right. \\
& { }^{3} b_{p, q, \lambda}^{r}(f):=\left\{\begin{array}{llc}
\left(\sum_{j=0}^{\infty} a^{j r q}\left(E_{a^{j}}(f)_{p, \lambda}\right)^{q}\right)^{\frac{1}{q}} & \text { if } & 1 \leq q<\infty, \\
\sup _{j \in Z_{+}} a^{j r} E_{a^{j}}(f)_{p, \lambda} & \text { if } & q=\infty,
\end{array}\right. \\
& { }^{4} b_{p, q, \lambda}^{r}(f):=\left\{\begin{array}{llc}
\inf \left(\sum_{j=0}^{\infty} a^{j r q}\left\|Q_{a^{j}}\right\|_{p, \lambda}^{q}\right)^{\frac{1}{q}} & \text { if } & 1 \leq q<\infty, \\
\inf \sup _{j \in Z_{+}} a^{j r}\left\|Q_{a^{j}}\right\|_{p, \lambda} & \text { if } & q=\infty .
\end{array}\right.
\end{aligned}
$$

The infimum is taken over all representations of $f$ in the form of series

$$
f(x)=\sum_{j=0}^{\infty} Q_{a^{j}}(x), Q_{a^{j}}(x) \in \mathfrak{M}\left(a^{j}, p, \lambda\right)
$$

convergent in $L_{p, \lambda}$. The ${ }^{j} B_{p, q, \lambda}^{r}$ are Banach spaces with respect to the norms

$$
\|f\|_{B_{p, q, \lambda}^{r}}:=\|f\|_{p, \lambda}+{ }^{j} b_{p, q, \lambda}^{r} .
$$

Theorem 5.1. The spaces ${ }^{j} B_{p, q, \lambda}^{r}, j=1,2,3,4$ coincide and their norms (5.19) are equivalent (that is, the Banach spaces ${ }^{j} B_{p, q, \lambda}^{r}$ are equivalent).

We mark that Theorem (1.5) follows from the equivalence of the Banach spaces ${ }^{1} B_{p, q, \lambda}^{r}$ and ${ }^{3} B_{p, q, \lambda}^{r}$. For brevity we use the notation ${ }^{j} B^{r}:={ }^{j} B_{p, q, \lambda}^{r},{ }^{j} b:={ }^{j} b_{p, q, \lambda}^{r}, E_{N}(f):=E_{N}(f)_{p, q, \lambda},\|f\|:=\|f\|_{p, \lambda}$ and so on. The expression $V_{1} \hookrightarrow V_{2}$ means that the Banach space $V_{1}$ is embedded in the Banach space $V_{2}$.

Proof of Theorem 5.1 is analogous of the Theorem 5.1 from [13]. 
Acknowledgments. The research of V.S. Guliyev and E.J. Ibrahimov is partially supported by the grant of Presidium Azerbaijan National Academy of Science 2015. The authors are thankful to the referee for very valuable comments.

\section{References}

1. P.L. Butzer, H. Behrens, Semi-groups of operators and approximation, Springer-Verlag, Berlin-Heidelberg-New York, 1967

2. V.I. Burenkov, H.V. Guliyev, Necessary and sufficient conditions for boundedness of the maximal operator in the local Morrey-type spaces, Dokl. Akad. Nauk, 2003, 391: 591-594

3. W.R. Bloom, Z. Xu, The Hardy-Littlewood maximal function for Chebli-Trimeche hypergroups, Contemp. Math., 1995, 183: 45-70

4. E.S. Belkina, Fourier-Dunkl harmonic analysis and approximation of functions: dis. of cand. of physical and mathematical siences, Petrozavodsk, 2008

5. W.C. Connett, A. L. Schwartz, A Hardy-Littlewood maximal inequality for Jacobi type hypergroups, Proc. Amer. Math. Soc., 1989, 106: 137-143

6. H. Chebli, Operateurs de translation generalisee et semi-groupes de convolution. In: Theorie du potentiel et analyse harmonique, Lecture Notes in Math., vol. 404, Springer- Verlag, Berlin, 1974, 35-59

7. R. Caroll, Transmutation, Scattering Theory and Special Functions, North-Holland, Amsterdam-New YorkOxford, 1982

8. J. Delsarte, Une extension nouvelle de la theorie des functions periodiques de Bohr, Acta Math., 1938, 69: 259-317

9. L. Durand, P.M. Fishbane, L.M. Simmons, Exspansion formulas and addition theorems for Gegenbayer functions, J. Math. Phys., 1976, 17: 1933-1948

10. I.M. Gelfand, G.E. Shilov, The Fourier transformation of the fast growing of functions and the questions of only solution of the problems Cauchy, Uspekhi Mat. Nauk, t. VIII, vip., 1953, 6: 1-54

11. V.S. Guliyev, Sobolev's theorem for B-Riesz potentials, Dokl. Akad. Nauk, 1998, 358: 450-451

12. V.S. Guliyev, Some properties of the anisotropic Riesz-Bessel potential, Anal. Math., 2000, 26: 99-118

13. V.S. Guliyev, M.N. Omarova, On fractional maximal function and fractional integral on the Laguerre hypergroup, J. Math. Anal. Appl., 2008, 340: 1058-1068

14. V.S. Guliyev, E.J. Ibrahimov, On equivalent normalizations of functional spaces associated with the generalized shift, Analysis Math., 2008, 34: 83-103

15. V.S. Guliyev, E.J. Ibrahimov, On estimating the approximation of locally summable functions by Gegenbauer singular integrals, Georgian Math. J., 2008, 15: 251-262

16. V.S. Guliyev, E.J. Ibrahimov, Calderon reproducing formula associated with the Gegenbauer operator on the half-line, J. Math. Anal. Appl., 2007, 335: 1079-1094

17. I.S. Gradshteyn, I.I. Rizhik, Table of Integrals, Summs, Series and Product, Moscow, 1971

18. E.J. Ibrahimov, On Gegenbauer transformation on the half-line, Georgian Math. J., 2011, 18: 497-515

19. V.S. Guliyev, E.J. Ibrahimov, Generalized Gegenbauer shift and some problems of the theory of approximation of functions on the metric $L_{2, \lambda}$. Trans. Natl. Acad. Sci. Azerb. Ser. Phys.-Tech. Math. Sci. , 2015, 36(2): 24-56

20. E.J. Ibrahimov, S.A. Jafarova, On approximation order of functions by Gegenbauer singular integrals, Proc. Inst. Math. Mech. Acad. Sci. Azerb., 2011, 35: 39-52

21. E.J. Ibrahimov, S.A. Jafarova, On convergence and convergence order of Gegenbauer $m$-singular integrals, Proc. A. Razmadze Math. Inst., 2012, 159: 21-42

22. Ar.S. Dzhafarov, Generalized moduli of continuity and their connections with best approximations. I. (Russian) Linear operators and their applications (Russian), 45-72, Azerbaidzhan. Gos. Univ., Baku, 1986.

23. I.A. Kipriyanov, The singulars of elliptic lasting problems, I: Nauka; Fizmatlit, 1997

24. J. Lofstrem, J. Peetre, Approximation theorems connected with generalized translations, Math. Ann., 1969, 181: 255-268

25. B.M. Levitan, The theory of the operators of generalized shift, I: Nauka, Fizmatlit, 1977

26. B.M. Levitan, Decomposition on the Bessel functions of Fourier series and integrals, Uspekhi Mat. Nauk, 1951, 6: 102-143

27. S.M. Nikolsky, Approximation of the functions of multiple variable and theorem of embedding, Nauka, M, 1977

28. S.M. Nikolsky, Generalization of one Bernstein's inequalities, Dokl. AN SSSR, 1977

29. S.S. Platonov, Bessel harmonic analysis and approximation of functions on the half-line, Izv. RAN. Ser. Mat., 2007, 71:11, 149-196

30. S.S. Platonov, Analogues of Bernstein's and Nikolskii's inequalities for one class of integer functions of exponential type, Dokl. Akad. Nauk, 2004, 398: 168-171 
31. S.S. Platonov, Fourier-Jacobi harmonic analysis and approximation of functions, Izv. Ross. Akad. Nauk Ser. Mat., 2014, 78:1, 117-166

32. S.S. Platonov, Generalized Bessel shift and some problems of the approximation theory of functions on the half-line, Sib. Math. Journ., 2009, 50: 154-174

33. M.K. Potapov, On approximation of the non-periodical functions by the algebraical polynomials, Vest. Moscow Univ. Mathem. Mechan., 1960, 4: 14-25

34. M.K. Potapov, On structural and constructive descriptions of some classes of functions, Proc. MIAN SSSR, 1974, 131: 211-231

35. M.K. Potapov, On approximation of function of functions by Jacobi polynomials, Vestn. Moscow Univ. Mathem. Mechan., 1977, 5: 70-82

36. M.K. Potapov, On approximation one operator of generalized shift on the approximation theory, Vestn. Moscov Univ. Mathem. Mechan., 1998, 3: 38-48

37. S.Z. Rafalson, On approximation of the functions of Fourier-Jacobi sums, Investiya Vuzov Mathem., 1968, 4: $54-62$

38. S.Z. Rafalson, On approximation of the functions in mean Fourier-Gegenbauer sums, Mathem. Zametki, 1968, 3: $587-596$

39. S.Z. Rafalson, Some direct and inverse theorems on approximation of functions in the metric of $L_{p, \alpha}$ by the algebraicals polynomials, Dep. VINITI, 1979

40. S.B. Stechkin, Generalization of some S. N. Bernstein's inequalities, Dokl. AN SSSR, 1948, 60: 1511-1514

41. A.F. Timan, Theory of approximation of functions of really variable, Fizmatgiz, M. 1960

42. K. Trimeche, Generalized harmonic analysis and wavelet packets, Gordon and Breach Sci. Publ., Amsterdam, 2000

43. A.P. Terekhin, Bounded groups of operators and best approximation, Differentials equations and calculating mathematics, Saratov: Publ. of Saratov University, 1975, 2: 3-28

44. Yong Ping Lee, Tshus Mey Su, V.I. Ivanov, Some problems of the approximation theory of functions in space $L_{p}$ on the line with weight degree, Mat. Zametki, 2011, 90: 362-383

45. G.V. Zhidkov, Constructive description of one class of non-periodical functions, DAN SSSR, 1966, 169: $1002-1005$ 